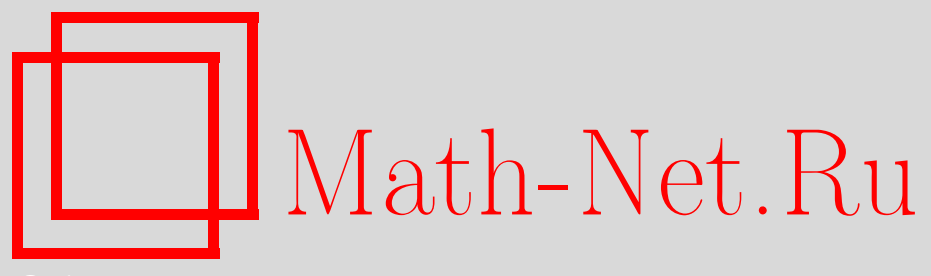

А. В. Домрин, Мероморфное продолжение решений солитонных уравнений, Изв. РАН. Сер. матем., 2010, том 74, выпуск $3,23-44$

DOI: https://doi.org/10.4213/im2784

Использование Общероссийского математического портала Math-Net.Ru подразумевает, что вы прочитали и согласны с пользовательским соглашением http://www . mathnet.ru/rus/agreement

Параметры загрузки:

IP : 54.237 .206 .68

26 апреля 2023 г., 15:20:10

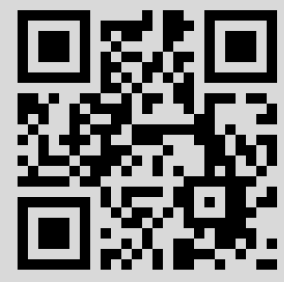


УДК $517.958+517.547 .24$

\title{
А. В. Домрин
}

\section{Мероморфное продолжение решений солитонных уравнений}

\begin{abstract}
Рассмотрены локальные варианты прямого и обратного преобразований рассеяния и описаны их аналитические свойства, аналогичные свойствам классических преобразований Лапласа и Бореля. Это позволяет изучать локальные голоморфные решения тех интегрируемых эволюционных уравнений на $\mathbb{C}_{x t}^{2}$, комплексифицированные формы которых задаются условием нулевой кривизны для связности вида $U d x+V d t$, где $U-$ линейная функция от спектрального параметра $z$, а $V$ - полином степени $m \geqslant 2$ от $z$. Показано, что локальная голоморфная задача Коши для таких уравнений разрешима тогда и только тогда, когда данные рассеяния начального условия принадлежат классу Жеврея $1 / m$. Показано также, что любое локальное голоморфное решение продолжается при каждом фиксированном $t$ до глобально мероморфной функции от $x$.
\end{abstract}

Библиография: 19 наименований.

Ключевые слова: солитонные уравнения, аналитическое продолжение.

\section{$\S 1$. Введение}

В настоящей работе изучается вопрос об аналитическом продолжении локально заданных решений интегрируемых эволюционных уравнений. Например, всякое решение $u(x, t)$ уравнения Кортевега-де Фриза

$$
u_{t}=k u u_{x}+l u_{x x x}
$$

(где $k, l \in \mathbb{C} \backslash\{0\}-$ произвольные константы), голоморфное в бидиске $\{(x, t) \in$ $\left.\mathbb{C}^{2}|| x-x_{0}\left|<\delta_{1},\right| t-t_{0} \mid<\delta_{2}\right\}$, всегда продолжается до функции, мероморфной в "полосе" $\left\{(x, t) \in \mathbb{C}^{2}|| t-t_{0} \mid<\delta_{2}\right\}$ (и, вообще говоря, не допускает аналитического продолжения ни в какую бо́льшую область). Доказательство опирается на необходимое условие разрешимости локальной голоморфной задачи Коши $u\left(x, t_{0}\right)=u_{0}(x)$ для уравнения (1) в терминах так называемых данных рассеяния (представляющих собой нелинейный аналог преобразования Лапласа) начального условия $u_{0}(x)$, а также на аналитические свойства отображения, восстанавливающего голоморфный росток $u_{0}(x)$ в точке $x_{0}$ по его данным рассеяния (это отображение есть нелинейный аналог преобразования Бореля). Чтобы мотивировать применяемый подход и обозначения, мы приводим в §2 описание того, во что превращаются обе составные части доказательства для

Работа выполнена при финансовой поддержке РФФИ (гранты № 08-01-00014, 08-01-00743), Программы Президента РФ "Поддержка ведущих научных школ" (грант НШ-3877.2008.1) и программы SFB/TR-12 (Германия).

(C) А. В. Домрин, 2010 
классической линейной ситуации. Читатель может перейти сразу к $\S 3$, где даются общие определения и формулировки результатов. Оставшаяся часть работы посвящена доказательствам этих результатов и комментариям.

\section{§ 2. Уравнение теплопроводности}

Рассмотрим задачу Коши

$$
u_{t}=u_{x x}, \quad u(x, 0)=u_{0}(x) .
$$

Для нее не выполнены предположения теоремы Коши-Ковалевской, и заключение этой теоремы тоже неверно: задача (2) не имеет (даже локальных) голоморфных решений для многих голоморфных ростков $u_{0}(x)$ в начале координат, например для $u_{0}(x)=\left(1+x^{2}\right)^{-1}$. Действительно, полагая $u_{0}(x)=\sum_{k=0}^{\infty} c_{k} x^{k}$ и пользуясь тем, что из (2) вытекают равенства $\partial_{t}^{k} u=\partial_{x}^{2 k} u$ для всех $k \geqslant 1$, можно записать

$$
u(0, t)=\sum_{k=0}^{\infty} \partial_{t}^{k} u(0,0) \frac{t^{k}}{k !}=\sum_{k=0}^{\infty} d_{k} c_{2 k} t^{k},
$$

где коэффициенты $d_{k}:=(2 k) ! / k$ ! весьма быстро возрастают при $k \rightarrow \infty$. Чтобы ряд для $u(0, t)$ имел ненулевой радиус сходимости, числа $c_{2 k}$ должны при $k \rightarrow \infty$ стремиться к нулю с такой же или большей скоростью. Точный результат (см., например, классический учебник [1, § 541]) состоит в том, что задача (2) имеет локальное голоморфное решение $u(x, t)$ в окрестности начала координат в $\mathbb{C}^{2}$ тогда и только тогда, когда росток $u_{0}(x)$ продолжается до целой функции порядка не выше 2 и конечного типа: $u_{0} \in \mathcal{O}(\mathbb{C})$ и существуют $A, B>0$ такие, что $\left|u_{0}(x)\right| \leqslant A \exp \left(B|x|^{2}\right)$ для всех $x \in \mathbb{C}$.

Если начальные данные $u_{0}(x)$ представляют собой целую функцию экспоненциального типа (т. е. $u_{0} \in \mathcal{O}(\mathbb{C})$ и выполнено неравенство $\left|u_{0}(x)\right| \leqslant A e^{R|x|}$ ), то задачу Коши (2) можно решить с помощью преобразования Лапласа (см., например, $\left[2\right.$, гл. I, § 5]). Под преобразованием Лапласа функции $u_{0}(x)$ обычно понимается функция

$$
\varphi_{0}(z)=L u_{0}(z):=\int_{0}^{\infty} e^{-x z} u_{0}(x) d x,
$$

определенная и голоморфная в полуплоскости $\operatorname{Re} z>R$. Однако в нашем случае функция $\varphi_{0}(z)$ допускает аналитическое продолжение в точку $z=\infty$, причем росток функции $u_{0}(x)$ в начале координат восстанавливается по ростку функции $\varphi_{0}(z)$ в точке $\infty$ посредством известного преобразования Бореля. Поэтому нам будет удобнее определить преобразование Лапласа $L$ и преобразование Бореля $B$ формулами

$$
L\left(\sum_{k \geqslant 0} c_{k} x^{k}\right)=\sum_{k \geqslant 0} \frac{k ! c_{k}}{z^{k+1}}, \quad B\left(\sum_{k \geqslant 0} \frac{\sigma_{k}}{z^{k+1}}\right)=\sum_{k \geqslant 0} \frac{\sigma_{k}}{k !} x^{k},
$$

где ряды по $x$ сходятся для всех $x \in \mathbb{C}$, а ряды по $z$ сходятся при $|z|>R$, включая точку $z=\infty$. Ясно, что равенства $\varphi_{0}=L u_{0}$ и $u_{0}=B \varphi_{0}$ эквивалентны друг другу. Применение преобразования Лапласа к задаче (2) превращает 
эту задачу в обыкновенное дифференциальное уравнение $\partial_{t} \varphi=\left\{z^{2} \varphi(t, z)\right\}_{-}$ с начальным условием $\varphi(0, z)=\varphi_{0}(z)$, где $\varphi(t, z):=L u(x, t)$, а через $\{\cdot\}_{-}$ обозначена отрицательная часть ряда Лорана: $\left\{\sum_{k \in \mathbb{Z}} a_{k} z^{k}\right\}_{-}=\sum_{k \leqslant-1} a_{k} z^{k}$. Здесь учтена формула дифференцирования оригинала:

$$
L\left(u_{x x}\right)(z)=z^{2} L u(z)-z u(0)-u_{x}(0) .
$$

Легко проверить, что указанное уравнение на $\varphi(t, z)$ имеет единственное решение. Это решение определено для всех $t \in \mathbb{C}$ и задается формулой $\varphi(t, z)=$ $\left\{\exp \left(t z^{2}\right) \varphi_{0}(z)\right\}_{-}$. Полагая теперь $u(x, t)=B \varphi(t, z)$, получаем единственное локальное голоморфное решение $u(x, t)$ задачи Коши $(2)$ в окрестности начала координат. В данном случае, когда начальное условие $u_{0}(x)$ предполагается целой функцией экспоненциального типа, решение $u(x, t)$ оказывается целой функцией на всем $\mathbb{C}^{2}$.

Заметим, что полученная формула $u(x, t)=B\left(\left\{\exp \left(t z^{2}\right) L u_{0}(z)\right\}_{-}\right)$для решения задачи Коши (2) справедлива и в самом общем случае локальной разрешимости этой задачи, т. е. в случае, когда $u_{0}(x)$ - целая функция порядка не выше 2 и конечного типа: хотя ряд $L u_{0}(z)$ может иметь нулевой радиус сходимости, выражение $\left\{\exp \left(t z^{2}\right) L u_{0}(z)\right\}_{-}$, тем не менее, задает корректно определенный формальный степенной ряд от $z^{-1}$ при всех $t \in \mathbb{C}$ с достаточно малым $|t|$, а преобразование Бореля (3) указанного выражения (при каждом таком $t$ ) есть корректно определенная целая функция от $x$ порядка не выше 2 и конечного типа. Отсюда вытекает, что всякое локальное голоморфное решение уравнения теплопроводности продолжается при каждом фиксированном $t$ до целой функции от $x$. Изучение таких решений в $[3$, гл. Х] основано именно на формуле $u(x, t)=B\left(\left\{\exp \left(t z^{2}\right) L u_{0}(z)\right\}_{-}\right)$, называемой там разложсением решения в ряд по тепловым полиномам.

Сформулируем соответствующие результаты для следующей задачи Коши, которая является естественным обобщением задачи (2):

$$
u_{t}=P\left(\partial_{x}\right) u, \quad u(x, 0)=u_{0}(x) .
$$

Здесь $P(s)=\beta s^{m}+\gamma_{1} s^{m-1}+\cdots+\gamma_{m}-$ произвольный полином степени $m \geqslant 2$ с коэффициентами $\beta \in \mathbb{C} \backslash\{0\}, \gamma_{1}, \ldots, \gamma_{m} \in \mathbb{C}$. Для каждого $\alpha>0$ обозначим через $\operatorname{Ent}_{\alpha}$ множество всех целых функций $u(x)=\sum_{k \geqslant 0} c_{k} x^{k}$ порядка не выше $\alpha$ и конечного типа, а через $\mathrm{Gev}_{\alpha}$ - множество всех формальных степенных рядов вида $\varphi(z)=\sum_{k \geqslant 0} \sigma_{k} z^{-(k+1)}$ таких, что ряд $\sum_{k \geqslant 0}(k !)^{-\alpha} \sigma_{k} x^{k}$ имеет ненулевой радиус сходимости.

ПРЕДЛОЖЕНИЕ 1. При любом вещественном $m>1$ формуль (3) задают взаимно однозначное соответствие между пространствами $\operatorname{Ent}_{m /(m-1)}$ u $\mathrm{Gev}_{1 / m}$. Это верно и при $m=1$, если под $\mathrm{Ent}_{\infty}$ понимать пространство $\mathcal{O}(0)$ всех голоморфных ростков в точке $x=0$.

ПреДЛОЖЕНИЕ 2. (А) Задача (4) имеет локалъное голоморфное решение $u(x, t)$ в окрестности точки $\left(x_{0}, t_{0}\right) \in \mathbb{C}^{2}$ тогда и толъко тогда, когда $u_{0} \in$ $\operatorname{Ent}_{m /(m-1)}\left(\right.$ или, эквивалентно, $\left.L u_{0} \in \mathrm{Gev}_{1 / m}\right)$.

(В) Всякое голоморбное решение $u(x, t)$ уравнения (4) в бидиске $\{(x, t) \in$ $\left.\mathbb{C}^{2}|| x-x_{0}\left|<\delta_{1},\right| t-t_{0} \mid<\delta_{2}\right\}$ аналитически продолюается до функиии, 
голоморфной в полосе $\left\{(x, t) \in \mathbb{C}^{2}|| t-t_{0} \mid<\delta_{2}\right\}$ и принадлежащей $\operatorname{Ent}_{m /(m-1)}$ как функиия от $x$ при каждом фиксированном $t$.

(C) Если в обозначениях утверждения (B) функиия $u_{0}(x):=u\left(x, t_{0}\right)$ nринадлежит Ent $_{\alpha}$ для некоторого $\alpha<m /(m-1)$, то решение $u(x, t)$ является целой функцией на всем $\mathbb{C}^{2}$.

Предложение 1 доказывается прямой проверкой с помощью хорошо известных формул, выражающих порядок и тип целой функции через ее коэффициенты Тейлора (см., например, [2]). Предложение 2,(А) следует, например, из доказываемой ниже теоремы 2, (А) (см. §3) с учетом предложения 1. Утверждения (В) и (С) предложения 2 вытекают из предложения 1 и формулы $u(x, t)=B\left(\left\{\exp (t P(z)) L u_{0}(z)\right\}_{-}\right)$для единственного локального голоморфного решения задачи (4). Заметим, что никаких утверждений об аналитическом продолжении такого решения по $t$ в общем случае сделать нельзя: если начальное условие $u(x, 0)=u_{0}(x)$ в (4) заменить на $\partial_{x}^{j} u(0, t)=v_{j}(t), j=0,1, \ldots, m-1$, то полученная система будет иметь единственное голоморфное решение в окрестности начала координат по теореме Коши-Ковалевской, так что росток $u(0, t)$ для локального голоморфного решения рассматриваемого уравнения можно выбрать произвольным. Однако предложение $2,(\mathrm{C})$ все же дает такое продолжение (до целой функции на всем $\mathbb{C}^{2}$ ) в том случае, когда начальная функция $u_{0}(x)$ задачи Коши (4) принадлежит не только пространству $\operatorname{Ent}_{m /(m-1)}$ (что необходимо и достаточно для локальной разрешимости), но и пространству $\operatorname{Ent}_{\alpha}$ при $\alpha<m /(m-1)$.

Оказывается, что значительная часть изложенных результатов переносится со случая линейных уравнений вида (4) на широкий класс нелинейных эволюционных уравнений, интегрируемых методом обратной задачи рассеяния. Этот класс включает в себя практически все изучаемые в [4]-[6] эволюционные уравнения, содержащие лишь первую производную по времени (например, в него входят уравнение Кортевега-де Фриза (1) и нелинейное уравнение Шрёдингера вместе с их иерархиями и модификациями, но не входит уравнение sine-Gordon). В следующем параграфе мы опишем этот класс и применяемый для его изучения метод обратной задачи рассеяния как матричное нелинейное обобщение уравнений (4) и преобразований (3).

\section{§ 3. Формулировки основных результатов}

Метод обратной задачи рассеяния для эволюционных интегрируемых уравнений имеет несколько вариантов (см. [4]-[6]), различающихся граничными условиями, которые налагаются на изучаемые решения. Мы пользуемся локальным вариантом, имеющим начало в [7], [8] и изучавшимся также в [9].

Сначала определим аналог преобразования $L$, введенного в (3). Пусть $\operatorname{gl}(n, \mathbb{C})$ - множество всех комплексных $(n \times n)$-матриц, $\mathcal{O}\left(x_{0}\right)$ - множество ростков всех голоморфных $\operatorname{gl}(n, \mathbb{C})$-значных отображений в точке $x_{0} \in \mathbb{C}$, а $\mathcal{O}\left(x_{0}\right)^{\text {od }}$ - множество всех внедиагональных ростков $q \in \mathcal{O}\left(x_{0}\right)$, т. е. таких, что $q_{l l}(x) \equiv 0$ при $l=1, \ldots, n$. Для каждого $\alpha \geqslant 0$ рассмотрим множество $\mathrm{Gev}_{\alpha}$ (называемое классом Жеврея $\alpha$ ) всех формальных степенных рядов вида $\varphi(z)=\sum_{k=0}^{\infty} \varphi_{k} z^{-(k+1)}, \varphi_{k} \in \operatorname{gl}(n, \mathbb{C})$, таких, что ряд $\sum_{k=0}^{\infty}(k !)^{-\alpha}\left|\varphi_{k}\right| x^{k}$ имеет 
ненулевой радиус сходимости. Здесь $|\cdot|$ означает любую норму на $\mathrm{gl}(n, \mathbb{C})$, обладающую свойством $|A B| \leqslant|A||B|$. Обозначим через $\operatorname{Gev}_{\alpha}^{\text {od }}$ множество всех внедиагональных $\varphi \in \operatorname{Gev}_{\alpha}$, т. е. таких, что $\left(\varphi_{k}\right)_{l l}=0$ для всех $k \geqslant 0$ и всех $l=1, \ldots, n$. Положим также $\operatorname{Gev}_{\alpha-0}:=\bigcup_{0 \leqslant s<\alpha} \operatorname{Gev}_{s}$.

Фиксируем произвольную диагональную матрицу $a \in \operatorname{gl}(n, \mathbb{C})$ с простым спектром (т. е. все ее собственные значения различны) и назовем локальнъми данными рассеяния любого внедиагонального голоморфного ростка $q \in$ $\mathcal{O}\left(x_{0}\right)^{\text {od }}$ формальный степенной ряд

$$
L q(z):=m\left(x_{0}, z\right)-I,
$$

где $m(x, z)=I+\sum_{k=0}^{\infty} m_{k}(x) z^{-(k+1)}$ - единственное решение дифференциального уравнения $m_{x}=(a z+q(x)) m-m a z$ в классе формальных степенных рядов указанного вида с $m_{k} \in \mathcal{O}\left(x_{0}\right)$ для всех $k \geqslant 0$ такое, что ряд $m\left(x_{0}, z\right)-I$ внедиагонален (существование и единственность такого решения доказаны ниже в 6$)$. Здесь и далее через $I$ обозначается единичная $(n \times n)$-матрица. С геометрической точки зрения $m(x, z)$ - формальное калибровочное преобразование диагональной связности $a z d x$ в связность $(a z+q(x)) d x$. Преобразование $L$ из (3) является частным случаем $(5)$ : если $q(x)-(2 \times 2)$-матрица с единственным ненулевым элементом $q_{12}(x)=u(x)$, разность $a_{11}-a_{22}$ диагональных элементов матрицы $a$ равна 1 и в качестве начальной точки выбирается $x_{0}=0$, то $L q(z)$ есть $(2 \times 2)$-матрица с единственным ненулевым элементом $(L q)_{12}(z)=-L u(z)$ (см. $[9, \S 6])$. Следующая теорема описывает аналитические свойства преобразования (5).

Tеорема 1. (A) Отображсение $q \mapsto L q$ есть биекиия $\mathcal{O}\left(x_{0}\right)^{\text {od }}$ на $\mathrm{Gev}_{1}^{\text {od }}$.

(B) Если $q \in \mathcal{O}\left(x_{0}\right)^{\text {od }}$ u $L q \in \mathrm{Gev}_{1-0}$, то росток $q(x)$ аналитически продолжается до мероморфной функиии на всем $\mathbb{C}$.

Теперь определим интересующий нас класс эволюционных уравнений. Фиксируем точку $x_{0} \in \mathbb{C}$. Отображение $F: \mathcal{O}\left(x_{0}\right) \rightarrow \mathcal{O}\left(x_{0}\right)$ называется дифференциальным полиномом, если для каждого $\kappa \in \mathcal{O}\left(x_{0}\right)$ функция $F(\kappa)$ есть обычный полином (один и тот же для всех $\kappa$ ) от функции $\kappa$ и ее производных $\kappa_{x}, \kappa_{x x}, \ldots$.

Пусть $a, b, c_{1}, c_{2}, \ldots \in \operatorname{gl}(n, \mathbb{C})$ - диагональные матрицы, причем $a$ и $b$ имеют простой спектр. Тогда существует единственная последовательность дифференциальных полиномов $F_{0}, F_{1}, F_{2}, \ldots$ такая, что $F_{0}(\kappa) \equiv b, F_{m}(0) \equiv c_{m}$ для всех $m \geqslant 1$, и формальный степенной ряд $F(\kappa, z):=\sum_{m=0}^{\infty} F_{m}(\kappa) z^{-m}$ удовлетворяет дифференциальному уравнению $\partial_{x} F(\kappa, z)=[a z+\kappa, F(\kappa, z)]$ тождественно по $\kappa \in \mathcal{O}\left(x_{0}\right)^{\text {od }}$ и по $z$ (см. [9, теорема 1]). Фиксируем целое число $m \geqslant 2$ и рассмотрим задачу Коши с начальным условием $q\left(x, t_{0}\right)=q_{0}(x)$ для уравнения

$$
q_{t}=\left[a, F_{m+1}(q)\right],
$$

где $q_{0} \in \mathcal{O}\left(x_{0}\right)^{\text {od }}$ - заданный внедиагональный голоморфный росток в точке $x_{0} \in \mathbb{C}$, а $q(x, t)$ - искомая внедиагональная голоморфная функция в окрестности точки $\left(x_{0}, t_{0}\right) \in \mathbb{C}^{2}$. С геометрической точки зрения уравнение $(6)$ есть условие нулевой кривизны $U_{t}-V_{x}+[U, V]=0$ для связности $U(x, t, z) d x+$ $V(x, t, z) d t$, где $U(x, t, z)=a z+q(x, t)$ и $V(x, t, z)=\sum_{j=0}^{m} F_{m-j}(q)(x, t) z^{j}$ суть полиномы от $z$ степеней 1 и $m$ соответственно. Представление интегрируемых 
систем в виде условий нулевой кривизны было впервые использовано в [10] (применительно к уравнению Кортевега-де Фриза), а после работы [7] стало одним из основных средств изучения таких систем (см. [4]-[6]).

Уравнения (6), отвечающие всевозможным наборам диагональных матриц $a, b, c_{1}, c_{2}, \ldots$, являются комплексифицированными формами интегрируемых эволюционных уравнений интересующего нас класса. Это означает, что для получения искомого эволюционного уравнения следует наложить на компоненты матрицы $q(x)$ дополнительные алгебраические условия, называемые редукциями, или условиями симметрии (см. их подробное обсуждение в [4]-[6]). Например, линейное уравнение (4) является частным случаем (6) при

$$
\begin{gathered}
a=\left(\begin{array}{cc}
1 / 2 & 0 \\
0 & -1 / 2
\end{array}\right), \quad b=\beta a, \\
c_{1}=\gamma_{1} a, \quad \ldots, \quad c_{m}=\gamma_{m} a, \quad q(x, t)=\left(\begin{array}{cc}
0 & u(x, t) \\
0 & 0
\end{array}\right),
\end{gathered}
$$

а уравнение Кортевега-де Фриза $u_{t}=u_{x x x}-6 u u_{x}$ (из которого заменой масштаба вдоль координатных осей $x, t$ легко получается общее уравнение (1)) является частным случаем уравнения (6) при

$$
a=b=\left(\begin{array}{cc}
1 / 2 & 0 \\
0 & -1 / 2
\end{array}\right), \quad c_{1}=c_{2}=\cdots=0, \quad q(x, t)=\left(\begin{array}{cc}
0 & u(x, t) \\
1 & 0
\end{array}\right), \quad m=3
$$

(см. [4, гл. I, §8] или $[9, \S 1$, примеры (C) и (E)]). Таким образом, результат о продолжении решений уравнения Кортевега-де Фриза (см. §1) является частным случаем утверждения (В) следующей теоремы.

Теорема 2. (А) Задача Коши $q\left(x, t_{0}\right)=q_{0}(x)$ для уравнения (6) имеет локальное голоморфное решение $q(x, t)$ в окрестности точки $\left(x_{0}, t_{0}\right) \in \mathbb{C}^{2}$ тогда и только тогда, когда $L q_{0} \in \mathrm{Gev}_{1 / m}$. Если такое решение $q(x, t)$ существует, то только одно.

(В) Всякое голоморфное решение $q(x, t)$ уравнения (6) в произвольном бидиске $\left\{(x, t) \in \mathbb{C}^{2}|| x-x_{0}\left|<\delta_{1},\right| t-t_{0} \mid<\delta_{2}\right\}$ аналитически продолжается до функции, мероморфной в полосе $\left\{(x, t) \in \mathbb{C}^{2}|| t-t_{0} \mid<\delta_{2}\right\}$. При этом существует голоморфное решение $q_{0}(x, t)$ уравнения (6) в указанном бидиске, не допускающее аналитического продолюения ни через одну граничную точку упомянутой полосы.

(C) Если в обозначениях утверждения (B) функция $q_{0}(x):=q\left(x, t_{0}\right)$ удовлетворяет $L q_{0} \in \mathrm{Gev}_{(1 / m)-0}$, то решение $q(x, t)$ аналитически продолжается до функиии, мероморфной на всем $\mathbb{C}^{2}$.

Как и в предложении 2, утверждение (C) теоремы 2 порождено вопросом о том, допускают ли локальные решения уравнения (6) аналитическое продолжение не только по пространственной переменной $x$, но и по временно́й переменной $t$. Ответ в общем случае отрицательный согласно второй части утверждения (B). Но утверждение $(\mathrm{C})$ дает положительный ответ для случая, когда $L q_{0} \in \bigcup_{\alpha<1 / m} \mathrm{Gev}_{\alpha}$. Условия утверждения (C) заведомо выполняются, если задача Коши с начальным условием $q_{0}(x)$ разрешима для некоторого старшего потока той же иерархии, т. е. для уравнения (6) с теми же матрицами $a$ 
и $b$, но с бо́льшим номером $m_{1}>m$. Дальнейшие комментарии, касающиеся формулировок и приложений теорем 1 и 2 , содержатся в $\S 9$.

\section{§4. Леммы о пространствах формальных степенных рядов}

Обратное преобразование рассеяния восстанавливает росток $q(x)$ по формальному степенному ряду $L q(z)$. Следуя подходу из работы [7], мы строим это преобразование с помощью задачи Римана о факторизации матричнозначных формальных рядов Лорана. Настоящий параграф содержит леммы, на основе которых в $\S 5$ будет установлена теорема о разрешимости указанной задачи Римана.

Пусть даны числа $\alpha \geqslant 0, m \geqslant 1$ и $A, B>0$. Рассмотрим множество $G_{\alpha}(A)$ всех формальных рядов $\varphi(z)=\sum_{k=0}^{\infty} \varphi_{k} z^{-(k+1)} \in \operatorname{Gev}_{\alpha}$ таких, что $\|\varphi\|_{\alpha, A}:=$ $\sum_{k=0}^{\infty}(k !)^{-\alpha}\left|\varphi_{k}\right| A^{k}<\infty$, и множество $E_{m}(B)$ всех целых $\operatorname{gl}(n, \mathbb{C})$-значных функций $\varepsilon(z)=\sum_{l=0}^{\infty} \varepsilon_{l} z^{l}$, коэффициенты которых удовлетворяют $\left|\varepsilon_{l}\right| \leqslant C(l !)^{-1 / m} B^{l}$ для всех $l$ и некоторой константы $C$. Ясно, что $G_{\alpha}(A)$ - банахово пространство с нормой $\|\cdot\|_{\alpha, A}$, изометрически изоморфное $l_{1}$, а $E_{m}(B)$ - банахово пространство с нормой $\|\varepsilon\|_{m, B}:=\sup _{l \geqslant 0}\left|\varepsilon_{l}\right|(l !)^{1 / m} B^{-l}$, изометрически изоморфное $l_{\infty}$. Через $\{\cdot\}_{+}$и $\{\cdot\}_{-}$будем обозначать положительную и отрицательную части формального ряда Лорана: $\left\{\sum_{k \in \mathbb{Z}} a_{k} z^{k}\right\}_{+}=\sum_{k \geqslant 0} a_{k} z^{k}$, $\left\{\sum_{k \in \mathbb{Z}} a_{k} z^{k}\right\}_{-}=\sum_{k \leqslant-1} a_{k} z^{k}$.

Лемма 1. Пусть $A>B>0, m \geqslant 1 u 0 \leqslant \alpha \leqslant 1 / m$. Тогда произведение элементов из $G_{\alpha}(A)$ и $E_{m}(B)$, взятых в любом порядке, есть корректно определенный ряд Лорана, принадлежащий $G_{\alpha}(A-B)+E_{m}(B)$. Отображения $(\varphi, \varepsilon) \mapsto\{\varphi \varepsilon\}_{ \pm} u(\varphi, \varepsilon) \mapsto\{\varepsilon \varphi\}_{ \pm}$являются непрерывными билинейными формами на $G_{\alpha}(A) \times E_{m}(B)$ со значениями в $G_{\alpha}(A-B)$ и $E_{m}(B)$.

ДоказАтельство. Имеем $\{\varphi \varepsilon\}_{-}=\sum_{k=0}^{\infty} \psi_{k} z^{-(k+1)}$ и $\{\varphi \varepsilon\}_{+}=\sum_{l=0}^{\infty} \delta_{l} z^{l}$, где $\psi_{k}=\sum_{p=0}^{\infty} \varphi_{k+p} \varepsilon_{p}$ и $\delta_{l}=\sum_{q=0}^{\infty} \varphi_{q} \varepsilon_{q+l+1}$. Обозначим $\|\varepsilon\|_{m, B}$ через $C$. Пользуясь неравенствами $m \geqslant 1,1 / m \geqslant \alpha$ и формулой бинома Ньютона, получаем

$$
\begin{aligned}
& \left\|\{\varphi \varepsilon\}_{-}\right\|_{\alpha, A-B}=\sum_{k=0}^{\infty} \frac{\left|\psi_{k}\right|(A-B)^{k}}{(k !)^{\alpha}} \leqslant \sum_{k, p=0}^{\infty} \frac{\left|\varphi_{k+p}\right|\left|\varepsilon_{p}\right|(A-B)^{k}}{(k !)^{\alpha}} \\
& \leqslant C \sum_{k, p=0}^{\infty}\left|\varphi_{k+p}\right| \frac{B^{p}(A-B)^{k}}{(k !)^{\alpha}(p !)^{1 / m}} \leqslant C \sum_{s=0}^{\infty} \frac{\left|\varphi_{s}\right|}{(s !)^{\alpha}} \sum_{k=0}^{s}\left(\frac{s !}{k !(s-k) !}\right)^{\alpha} B^{s-k}(A-B)^{k} \\
& \leqslant C \sum_{s=0}^{\infty} \frac{\left|\varphi_{s}\right|}{(s !)^{\alpha}}(B+A-B)^{s}=C\left\|_{\varphi}\right\|_{\alpha, A}=\|\varphi\|_{\alpha, A}\|\varepsilon\|_{m, B},
\end{aligned}
$$

откуда и вытекают все требуемые свойства $\{\varphi \varepsilon\}_{-}$. Доказательство для $\{\varepsilon \varphi\}_{-}$ аналогично.

С другой стороны, с помощью очевидного неравенства $q$ ! $(l+1) ! \leqslant(q+l+1)$ ! и условия $B<A$ получаем

$$
\left|\delta_{l}\right|=\left|\sum_{q=0}^{\infty} \varphi_{q} \varepsilon_{q+l+1}\right| \leqslant C \sum_{q=0}^{\infty} \frac{\left|\varphi_{q}\right| B^{q+l+1}}{((q+l+1) !)^{1 / m}} \leqslant \frac{C B^{l+1}}{((l+1) !)^{1 / m}} \sum_{q=0}^{\infty} \frac{\left|\varphi_{q}\right| A^{q}}{(q !)^{1 / m}},
$$


откуда $\left\|\{\varphi \varepsilon\}_{+}\right\|_{m, B} \leqslant B\|\varphi\|_{1 / m, A}\|\varepsilon\|_{m, B} \leqslant B\|\varphi\|_{\alpha, A}\|\varepsilon\|_{m, B}$. Этим доказаны все требуемые свойства $\{\varphi \varepsilon\}_{+}$(и, аналогично, $\left.\{\varepsilon \varphi\}_{+}\right)$.

Лемма 2. Пусть $\alpha \geqslant 0$ и $A>0$. Тогда $\|\varphi \psi\|_{\alpha, A} \leqslant A\|\varphi\|_{\alpha, A}\|\psi\|_{\alpha, A}$ для всех $\varphi, \psi \in G_{\alpha}(A)$. Кроме того, множество $I+\mathrm{Gev}_{\alpha}$ является группой относительно обычного произведения формальных степенных рядов.

ДоказАТЕЛЬство. Выражение

$$
\|\varphi \psi\|_{\alpha, A}=\sum_{s=1}^{\infty} A^{s}(s !)^{-\alpha}\left|\sum_{k=0}^{s-1} \varphi_{k} \psi_{s-1-k}\right| \leqslant \sum_{k, l=0}^{\infty}\left|\varphi_{k}\right|\left|\psi_{l}\right| A^{k+l+1}((k+l+1) !)^{-\alpha}
$$

не превосходит выражения

$$
A\|\varphi\|_{\alpha, A}\|\psi\|_{\alpha, A}=\sum_{k, l=0}^{\infty}\left|\varphi_{k}\right|\left|\psi_{l}\right| A^{k+l+1}(k ! l !)^{-\alpha}
$$

так как $(k+l+1)$ ! $\geqslant k ! l !$. Этим доказана первая часть леммы, а также замкнутость множества $I+\mathrm{Gev}_{\alpha}$ относительно умножения. Осталось проверить, что $(I+\varphi)^{-1} \in I+\operatorname{Gev}_{\alpha}$ для любого $\varphi \in \operatorname{Gev}_{\alpha}$. Однако если $\varphi(z)=\sum_{j=1}^{\infty} \varphi_{j} z^{-j}$, то $(I+\varphi(z))^{-1}-I=\sum_{j=0}^{\infty} \psi_{j} z^{-j}$, где коэффициенты $\psi_{1}=-\varphi_{1}, \psi_{2}=-\left(\psi_{1} \varphi_{1}+\varphi_{2}\right), \ldots$ удовлетворяют неравенствам $\left|\psi_{j}\right| \leqslant$ $\sum_{s=1}^{j-1}\left|\psi_{j-s}\right|\left|\varphi_{s}\right|+\left|\varphi_{j}\right|$ при всех $j \geqslant 1$. Поскольку $(s !)^{\alpha}((j-s) !)^{\alpha} \leqslant(j !)^{\alpha}$ для всех целых $s=1, \ldots, j$, эти неравенства можно переписать в виде одного неравенства $2 M(R) \leqslant M(R) L(R)+1$ для формальных степенных рядов $L(R):=1+\sum_{s=1}^{\infty}(s !)^{-\alpha}\left|\varphi_{s}\right| R^{s}$ и $M(R):=1+\sum_{t=1}^{\infty}(t !)^{-\alpha}\left|\psi_{t}\right| R^{t}$. По условию ряд $L(R)$ сходится для всех достаточно малых $R>0$, причем $L(0)=1$. Но тогда ряд $M(R) \leqslant 1 /(2-L(R))$ также сходится для всех достаточно малых $R>0$, что и требовалось доказать.

Лемма 3. Если $0 \leqslant \alpha_{1}<\alpha_{2}$, то $G_{\alpha_{1}}\left(A_{1}\right) \subset G_{\alpha_{2}}\left(A_{2}\right)$ для всех $A_{1}, A_{2}>0$. Если $A_{1}>A_{2}$, то $G_{\alpha}\left(A_{1}\right) \subset G_{\alpha}\left(A_{2}\right)$ для всех $\alpha \geqslant 0$. Если $1 \leqslant m_{1}<m_{2}$, то $E_{m_{1}}\left(B_{1}\right) \subset E_{m_{2}}\left(B_{2}\right)$ для всех $B_{1}, B_{2}>0$. Если $B_{1}<B_{2}$, mо $E_{m}\left(B_{1}\right) \subset E_{m}\left(B_{2}\right)$ для всех $m \geqslant 1$. Все эти вложения банаховых пространств непрерывны.

ДокАЗАТЕЛЬСтво вытекает непосредственно из определений.

Лемма 4. Пусть функиии $E(z)=\sum_{p=0}^{\infty} c_{p} z^{p}$ и $F(z)=\sum_{q=0}^{\infty} d_{q} z^{q}$ принадлежат $E_{m}(B)$, причем $E(z) F(z) \equiv I$. Тогда для всех $\varphi \in G_{1 / m}(A), A>2 B$, справедливо равенство $\left\{\{\varphi E\}_{-} F\right\}_{-}=\varphi$.

ДокАзАтЕльство. Согласно первой формуле из начала доказательства леммы 1 , коэффициент при $z^{-(k+1)}$ в степенном ряде $\left\{\{\varphi(z) E(z)\}_{-} F(z)\right\}_{-}$ имеет вид

$$
\sum_{q=0}^{\infty}\left(\sum_{p=0}^{\infty} \varphi_{k+q+p} c_{p}\right) d_{q}=\sum_{r=0}^{\infty} \varphi_{k+r} \sum_{p+q=r} c_{p} d_{q}=\sum_{r=0}^{\infty} \varphi_{k+r} \delta_{0 r}=\varphi_{k}
$$

причем все преобразования законны в силу абсолютной сходимости. 
Лемма 5. Пусть $P(z)=\sum_{k=0}^{m} p_{k} z^{k}$ - полином степени не выше $m$ с коэ фициентами из $\mathrm{gl}(n, \mathbb{C})$. Тогда бункиия $e^{P(z)}$ принадлежст $E_{m}(B)$ при любом $B>\left(e m\left|p_{m}\right|\right)^{1 / m}$.

ДокАЗАтЕльСтво. Для любого $\beta>\left|p_{m}\right|$ имеем $|P(z)| \leqslant \beta|z|^{m}$ при $|z| \geqslant R_{0}$. Поэтому из неравенств Коши вытекает, что коэффициенты $\varepsilon_{l}$ разложения $e^{P(z)}=\sum_{l=0}^{\infty} \varepsilon_{l} z^{l}$ удовлетворяют неравенству $\left|\varepsilon_{l}\right| \leqslant R^{-l} \exp \left\{\beta R^{m}\right\}$ для всех $R \geqslant R_{0}$. Минимум правой части последнего неравенства как функции от $R$ достигается при $R=(l /(m \beta))^{1 / m}$, что не меньше $R_{0}$ при $l \geqslant l_{0}$. Следовательно, при $l \geqslant l_{0}$ можно записать $\left|\varepsilon_{l}\right| \leqslant(e m \beta / l)^{l / m}$. Пользуясь очевидным неравенством $l ! \leqslant l^{l}$, получаем $\left|\varepsilon_{l}\right| \leqslant(l !)^{-1 / m} B^{l}$ при любом $B \geqslant(\text { em } \beta)^{1 / m}$, что и требовалось доказать.

\section{§ 5. Задача Римана}

Следующий результат играет ключевую роль в доказательствах теорем 1, 2 . Его неформальное описание таково. Пусть матричнозначный ряд $f(z)$ по степеням $1 / z$ со свободным членом $I$ принадлежит такому классу Жеврея, что произведение в левой части равенства (7) имеет смысл по леммам 1,2 . Утверждается, что если при $\xi=0$ это произведение можно записать с помощью множителей такого же типа, но взятых в обратном порядке, то такая запись возможна и в некоторой окрестности точки $\xi=0$, причем решение $\gamma_{ \pm}(\xi, z)$ голоморфно по $\xi$ в этой окрестности. Если $f$ принадлежит строго меньшему классу Жеврея, то указанная запись возможна всюду вне некоторой комплексной гиперповерхности, а решение $\gamma_{ \pm}(\xi, z)$ глобально мероморфно по $\xi$.

Теорема 3. (А) Пусть $N, m \geqslant 1$ - целье числа, а $P(\xi, z)$ - полином от $z$ степени не выше $m$, задающий голоморфное отображение $P: \mathbb{C}^{N} \times \mathbb{C} \rightarrow$ $\operatorname{gl}(n, \mathbb{C})$, причем $P(0, z) \equiv 0$. Тогда для любого ряда $f \in I+\mathrm{Gev}_{1 / m}$ найдутся окрестность $\Omega(f)$ начала координат в $\mathbb{C}^{N}$, числа $A, B>0$ и голоморфные отображения $\gamma_{-}: \Omega(f) \rightarrow I+G_{1 / m}(A)$ и $\gamma_{+}: \Omega(f) \rightarrow E_{m}(B)$ такие, что при каждом $\xi \in \Omega(f)$ выполнено следующее равенство формальных рядов Лорана:

$$
e^{P(\xi, z)} f^{-1}(z)=\gamma_{-}^{-1}(\xi, z) \gamma_{+}(\xi, z) .
$$

(В) Если в условиях утверждения (А) имеем $f \in I+\operatorname{Gev}_{(1 / m)-0}$, то существует целая функция $\tau_{f} \in \mathcal{O}\left(\mathbb{C}^{N}\right), \tau_{f}(0)=1$, такая, что при любом $A>0$ отображения $\tau_{f}(\xi)\left(\gamma_{-}(\xi, z)-I\right) u \tau_{f}(\xi)\left(\gamma_{-}^{-1}(\xi, z)-I\right)$ продолжаются до голоморбных отображений $\mathbb{C}^{N} \rightarrow G_{1 / m}(A)$. При этом для любого $R>0$ найдется $B(R)>0$ такое, что отображения $\tau_{f}(\xi) \gamma_{+}(\xi, z) u \tau_{f}(\xi) \gamma_{+}^{-1}(\xi, z)$ продолжаются в полидиск $D_{R}=\left\{\xi \in \mathbb{C}^{N}|| \xi_{1}|<R, \ldots,| \xi_{N} \mid<R\right\}$ до голоморфных отображений $D_{R} \rightarrow E_{m}(B(R))$. Кроме того, равенство (7) выполняется для всех значений $\xi \in \mathbb{C}^{N}$, удовлетворяющих $\tau_{f}(\xi) \neq 0$.

ДокАЗАТЕЛЬСтво. Положим $\varphi_{0}(z):=f^{-1}(z)-I$. По условию и лемме 2 имеем $\varphi_{0} \in G_{\alpha}\left(A_{0}\right)$ для некоторых $\alpha \in[0,1 / m]$ и $A_{0}>0$. С другой стороны, так как $P(0, z) \equiv 0$, то по лемме 5 для любого $B>0$ найдется $\delta(B)>0$ такое, что целая функция $e^{P(\xi, z)}$ принадлежит $E_{m}(B)$ при всех $\xi \in \mathbb{C}^{N}$ с условием $|\xi|:=\max \left(\left|\xi_{1}\right|, \ldots,\left|\xi_{N}\right|\right)<\delta(B)$. Выбирая $0<B<A_{0}$, получаем по лемме 1 , 
что степенные ряды $\left\{e^{P} f^{-1}\right\}_{-}=\left\{e^{P} \varphi_{0}\right\}_{-} \in G_{\alpha}\left(A_{0}-B\right)$ и $\left\{e^{P} f^{-1}\right\}_{+} \in E_{m}(B)$ корректно определены при всех таких $\xi$.

Фиксируем одно из таких значений $\xi$ и будем искать решение $\gamma_{-}(\xi, z)$ уравнения (7) в виде $\gamma_{-}=I+\varphi \in I+G_{\alpha}\left(A_{1}\right)$ для некоторого пока не определенного $A_{1}>0$. Соответствующее условие на $\varphi$ состоит в том, что

$$
\left\{(I+\varphi) e^{P} f^{-1}\right\}_{-}=0 .
$$

Это условие имеет вид системы линейных уравнений $\left(M T_{P}+K_{P}\right) \varphi=v_{P}$ с вектором

$$
v_{P}:=-\left\{e^{P} \varphi_{0}\right\}_{-} \in G_{\alpha}\left(A_{0}-B\right)
$$

в правой части и операторами

$$
M \varphi:=\varphi f^{-1}, \quad T_{P} \varphi:=\left\{\varphi e^{P}\right\}_{-}, \quad K_{P} \varphi:=\left\{\left\{\varphi e^{P}\right\}_{+} \varphi_{0}\right\}_{-}
$$

в левой части. Оператор $M: G_{\alpha}(A) \rightarrow G_{\alpha}(A)$ непрерывен и обратим для любого положительного $A \leqslant A_{0}$ (см. лемму 2 ), причем обратный оператор есть $M^{-1} \varphi=\varphi f$. Оператор $T_{P}: G_{\alpha}(A) \rightarrow G_{\alpha}(A-B)$ непрерывен (по лемме 1 ) и "почти обратим" (по лемме 4) в том смысле, что $T_{P} T_{-P} \varphi=T_{-P} T_{P} \varphi=\varphi$ для всех $\varphi \in G_{\alpha}(A)$ при $A>2 B$. Поэтому для нахождения нужного нам решения $\varphi$ соотношения (8) достаточно установить существование такого $\varphi \in G_{\alpha}\left(A_{1}\right)$, что

$$
\left(I+T_{-P} M^{-1} K_{P}\right) \varphi=T_{-P} M^{-1} v_{P}
$$

Следующая лемма показывает, что оператор $K_{P}$ может непрерывно отображать $G_{\alpha}\left(A_{1}\right)$ в $G_{\alpha}\left(A_{2}\right)$ для $A_{2}>A_{1}$. Это позволяет рассматривать левую часть (11) как непрерывный линейный оператор, действующий из некоторого банахова пространства в себя, и изучать обратимость этого оператора.

Лемма 6. Если $2^{\alpha} B<A_{1}<A_{0} u 0<2^{1+\alpha} A_{2}<A_{0}$, то $K_{P}$ является непрерывным и даже компактным линейным оператором из $G_{\alpha}\left(A_{1}\right)$ в $G_{\alpha}\left(A_{2}\right)$. При $0 \leqslant \alpha<1 / m$ это утверждение остается верным, если заменить неравенства $2^{\alpha} B<A_{1}<A_{0}$ на более слабое условие $0<A_{1}<A_{0}$.

ДокАЗАтельство. Для компактности оператора $K: l_{1} \rightarrow l_{1}$, имеющего в стандартном базисе $\left\{e_{0}, e_{1}, e_{2}, \ldots\right\}$ матрицу $\left(K_{p q}\right)_{p, q=0}^{\infty}$ (т. е. такого, что равенство $K x=y$ для финитных векторов $x=\sum_{q=0}^{\infty} x_{q} e_{q}$ и $y=\sum_{p=0}^{\infty} y_{p} e_{p}$ эквивалентно системе $\left.y_{p}=\sum_{q=0}^{\infty} K_{p q} x_{q}\right)$, достаточно выполнение неравенства $\sum_{p, q=0}^{\infty}\left|K_{p q}\right|<\infty$. (Действительно, в этом случае известная формула $\|X\|=\sup _{q \geqslant 0}\left\|X e_{q}\right\|=\sup _{q \geqslant 0} \sum_{p=0}^{\infty}\left|X_{p q}\right|$ для нормы оператора $X \in \mathcal{B}\left(l_{1}\right)$ показывает, что $K=\lim _{j \rightarrow \infty} \pi_{j} K$ является пределом конечномерных операторов по указанной норме, где $\pi_{j} \in \mathcal{B}\left(l_{1}\right)$ - проектор на $\left\langle e_{0}, e_{1}, \ldots, e_{j}\right\rangle$.) Проверим, что это неравенство выполняется для оператора $K:=i_{2} K_{P} i_{1}^{-1}$, где через $i_{s}: G_{\alpha}\left(A_{s}\right) \rightarrow l_{1}, s=1,2$, обозначены стандартные диагональные изометрии

$$
i_{s}\left(\sum_{k=0}^{\infty} \varphi_{k} z^{-(k+1)}\right)=\sum_{k=0}^{\infty}(k !)^{-\alpha}\left(A_{s}\right)^{k} \varphi_{k} e_{k} .
$$


Записывая $e^{P(\xi, z)}=\sum_{l=0}^{\infty} \varepsilon_{l} z^{l}$ и $\varphi_{0}(z)=\sum_{k=0}^{\infty} \varphi_{k}^{0} z^{-(k+1)}$, получаем для матричных элементов оператора $K$ формулу

$$
K_{p q}=\sum_{r=0}^{\infty} \frac{A_{2}^{p}}{(p !)^{\alpha}} \frac{(q !)^{\alpha}}{A_{1}^{q}} \varepsilon_{q+r+1} \varphi_{p+r}^{0} .
$$

По условию имеем $\left|\varepsilon_{l}\right| \leqslant C_{1}(l !)^{-1 / m} B^{l}$ и $\left|\varphi_{k}^{0}\right| \leqslant C_{2}(k !)^{\alpha}\left(A_{0}\right)^{-k}$ для некоторых констант $C_{1}, C_{2}>0$. Кроме того, очевидные неравенства $1 \leqslant C_{p+q+r}^{q}$ и $C_{p+q+r}^{p} \leqslant 2^{p+q+r}$ для биномиальных коэффициентов дают

$$
\frac{(q !)^{\alpha}((p+r) !)^{\alpha}}{(p !)^{\alpha}((q+r+1) !)^{1 / m}}=\frac{(q+r+1)^{-1 / m}}{((q+r) !)^{(1 / m)-\alpha}}\left(\frac{C_{p+q+r}^{p}}{C_{p+q+r}^{q}}\right)^{\alpha} \leqslant \frac{2^{(p+q+r) \alpha}}{((q+r) !)^{(1 / m)-\alpha}} .
$$

Пользуясь всеми этими оценками, получаем

$$
\begin{aligned}
\frac{1}{C_{1} C_{2}} \sum_{p, q=0}^{\infty}\left|K_{p q}\right| & \leqslant \sum_{p, q, r=0}^{\infty} \frac{A_{2}^{p} B^{q+r+1} 2^{(p+q+r) \alpha}}{A_{1}^{q} A_{0}^{p+r}((q+r) !)^{(1 / m)-\alpha}} \\
& =\sum_{p, q, r=0}^{\infty}\left(\frac{2^{\alpha} A_{2}}{A_{0}}\right)^{p}\left(\frac{2^{\alpha} B}{A_{1}}\right)^{q+r}\left(\frac{A_{1}}{A_{0}}\right)^{r} \frac{B}{((q+r) !)^{(1 / m)-\alpha}} .
\end{aligned}
$$

Обозначим число $\frac{2^{\alpha} B}{A_{1}}$ через $R$. Тогда неравенства из условия леммы показывают, что последний ряд не превосходит

$$
\sum_{p, q, r=0}^{\infty}\left(\frac{1}{2}\right)^{p} \frac{B R^{q+r}}{((q+r) !)^{(1 / m)-\alpha}}=\sum_{q, r=0}^{\infty} \frac{2 B R^{q+r}}{((q+r) !)^{(1 / m)-\alpha}}=2 B \sum_{s=0}^{\infty} \frac{(s+1) R^{s}}{(s !)^{(1 / m)-\alpha}} .
$$

Если $\alpha=1 / m$, то этот ряд сходится при $0<R<1$. Если $\alpha<1 / m$, то он сходится при всех $R>0$. Лемма доказана.

Лемма 7. В условиях леммы 6 операторы $K_{P}, T_{P}$ и вектор $v_{P}$ являются голоморфными в некоторой окрестности начала координат функииями от $\xi$ со значениями в банаховых пространствах $\mathcal{B}\left(G_{\alpha}\left(A_{1}\right), G_{\alpha}\left(A_{2}\right)\right), \mathcal{B}\left(G_{\alpha}\left(A_{2}\right)\right.$, $\left.G_{\alpha}\left(A_{2}-B\right)\right)$ и $G_{\alpha}\left(A_{0}-2 B\right)$ соответственно.

ДокАЗАтельство. Запишем $P(\xi, z)=\sum_{k=0}^{m} p_{k}(\xi) z^{k}$ для некоторых голоморфных функций $p_{0}, p_{1}, \ldots, p_{m}: \mathbb{C}^{N} \rightarrow \operatorname{gl}(n, \mathbb{C})$ и выберем окрестность $\Omega$ начала координат в $\mathbb{C}^{N}$ так, что все функции $p_{k}, k=0,1, \ldots, m$, ограничены в $\Omega$ и $\sup _{\xi \in \Omega}\left(e m\left|p_{m}(\xi)\right|\right)^{1 / m}<B / 2$. (Такая окрестность существует, поскольку $p_{k}(0)=0$ для всех $k$.) Тогда для коэффициентов $\varepsilon_{l}(\xi)$ разложения $e^{P(\xi, z)}=\sum_{l=0}^{\infty} \varepsilon_{l}(\xi) z^{l}$ имеем согласно доказательству леммы 5 оценку $\left|\varepsilon_{l}(\xi)\right| \leqslant(l !)^{-1 / m}(B / 2)^{l}$ для всех $\xi \in \Omega$ и $l \geqslant l_{0}(\xi)$, причем в силу ограниченности всех $p_{k}(\xi)$ число $l_{0}(\xi)$ может быть выбрано одним и тем же для всех $\xi \in \Omega$. Отсюда

$$
\left\|\sum_{l=l_{1}}^{\infty} \varepsilon_{l}(\xi) z^{l}\right\|_{m, B}=\sup _{l \geqslant l_{1}}\left|\varepsilon_{l}(\xi)\right|(l !)^{1 / m} B^{-l} \leqslant\left(\frac{1}{2}\right)^{l_{1}}, \quad l_{1} \geqslant l_{0} .
$$

Таким образом, ряд $\sum_{l=0}^{\infty} \varepsilon_{l}(\xi) z^{l}$ сходится к $e^{P(\xi, z)}$ равномерно на $\Omega$ по норме пространства $E_{m}(B)$. Подставляя этот ряд в $(9)$ для $v_{P}$ и в $(10)$ для $T_{P}$ 
и пользуясь оценками билинейных форм из леммы 1, получаем представление $T_{P}$ и $v_{P}$ в виде равномерного на $\Omega$ предела частичных сумм по норме соответствующих пространств. Эти частичные суммы голоморфны на $\Omega$, так как они являются конечными линейными комбинациями фиксированных элементов со скалярными коэффициентами, голоморфно зависящими от $\xi \in \Omega$. Поэтому их предел голоморфен на $\Omega$ по теореме Вейерштрасса. Этим утверждение леммы доказано для случаев $T_{P}$ и $v_{P}$.

Рассмотрим случай $K_{P}$. Из доказательства леммы 6 видно, что $K_{P}$ - равномерный на $\Omega$ предел по норме пространства $\mathcal{B}\left(G_{\alpha}\left(A_{1}\right), G_{\alpha}\left(A_{2}\right)\right)$ частичных сумм двойного ряда из конечномерных операторов. Каждый из этих операторов имеет матрицу с единственным ненулевым элементом $K_{p q}$ для некоторых $p, q$. Пользуясь формулой (12) и первой частью доказательства настоящей леммы, получаем, что каждый из этих операторов голоморфно зависит от $\xi \in \Omega$. Отсюда по теореме Вейерштрасса вытекает голоморфность оператора $K_{P}$ на $\Omega$ относительно указанной нормы. Лемма доказана.

Обозначим левую часть равенства (11) через $X(\xi) \varphi$, а правую - через $u(\xi)$. Пусть числа $B, A_{1}, A_{2}$ выбраны так, что $0<2 B<A_{1} \leqslant A_{2}-B$ и $4 A_{2}<A_{0}$ (например, можно положить $B=A_{0} / 16, A_{1}=5 A_{0} / 32$ и $A_{2}=7 A_{0} / 32$ ). Тогда из леммы 6 и непрерывности операторов $M$ и $T_{-P}$ (см. формулу (11)) вытекает, что при $|\xi|<\delta(B)$ оператор $X(\xi)$ есть непрерывный линейный оператор на банаховом пространстве $G_{\alpha}\left(A_{1}\right)$, а $u(\xi)$ является вектором из этого пространства. Поскольку оператор $X(\xi)$ непрерывен по $\xi$ (лемма 7 ) и обратим при $\xi=0$ (будучи тождественным), он обратим при всех $\xi$ в некоторой окрестности начала координат. Обозначим эту окрестность через $\Omega(f)$. Тогда при каждом $\xi \in \Omega(f)$ уравнение (11) имеет единственное решение $\varphi=X(\xi)^{-1} u(\xi) \in G_{\alpha}\left(A_{1}\right)$. По лемме 7 это решение задает голоморфное отображение $\varphi: \Omega(f) \rightarrow G_{\alpha}\left(A_{1}\right)$. Положим $\gamma_{-}:=I+\varphi$ и $\gamma_{+}:=\gamma_{-} e^{P} f^{-1}$. Тогда при каждом $\xi \in \Omega(f)$ выполнено равенство (7), причем формальный ряд

$$
\begin{aligned}
\gamma_{+} & =\left\{(I+\varphi) e^{P}\left(I+\varphi_{0}\right)\right\}_{+}=\left\{\varphi e^{P}\right\}_{+}+\left\{e^{P} \varphi_{0}\right\}_{+}+\left\{\varphi e^{P} \varphi_{0}\right\}_{+} \\
& =\left\{\varphi e^{P}\right\}_{+}+\left\{e^{P} \varphi_{0}\right\}_{+}+\left\{\left\{\varphi e^{P}\right\}_{+} \varphi_{0}\right\}_{+}
\end{aligned}
$$

принадлежит пространству $E_{m}(B)$ по лемме 1 . В доказательстве леммы 7 мы видели, что $\xi \mapsto e^{P(\xi, \cdot)}$ - голоморфное отображение из $\Omega(f)$ в $E_{m}(B)$. Пользуясь этим фактом, а также оценками билинейных форм из леммы 1 и установленной выше голоморфностью отображения $\varphi: \Omega(f) \rightarrow G_{\alpha}\left(A_{1}\right)$, получаем, что отображение $\gamma_{+}: \Omega(f) \rightarrow E_{m}(B)$ голоморфно. Утверждение (А) доказано.

Доказательство утверждения (В) проходит по той же схеме, что и выше. В нем используется следующая лемма о мероморфности семейства, обратного к голоморфному семейству фредгольмовых операторов индекса 0. Эту лемму следует считать хорошо известной. Очень близкие к ней утверждения содержатся, например, в [11] или [12]. Для полноты изложения мы приведем набросок доказательства.

ЛЕмма 8. Пусть $Y: \Omega \rightarrow \mathcal{B}(E)$ - голоморфное отображение полидиска $\Omega=$ $\left\{\xi \in \mathbb{C}^{N}|| \xi \mid<R\right\}$ в пространство линейных непрерывных операторов на банаховом пространстве $E$, причем операторы $Y(\xi)$ компактны для всех $\xi \in \Omega$ 
и оператор $I+Y\left(\xi_{0}\right)$ обратим для некоторой точки $\xi_{0} \in \Omega$. Тогда найдется голоморфная функция $\tau \in \mathcal{O}(\Omega), \tau\left(\xi_{0}\right)=1$, такая, что:

(i) $I+Y(\xi)$ обратим для тех и только тех $\xi \in \Omega$, для которых $\tau(\xi) \neq 0$;

(ii) $\tau(\xi)(I+Y(\xi))^{-1}$ - голоморфное отображение $\Omega \rightarrow \mathcal{B}(E)$.

ДокАЗАТЕЛЬСтво. Положим $X(\xi)=I+Y(\xi)$ и обозначим через $Z$ множество всех тех значений $\xi_{1} \in \Omega$, для которых оператор $X\left(\xi_{1}\right)$ не обратим, т. е. $0 \in \operatorname{sp} X\left(\xi_{1}\right)$. Из свойств спектра компактного оператора ясно, что 0 является изолированным собственным значением конечной кратности для оператора $X\left(\xi_{1}\right)$. Если $P$ - соответствующий спектральный проектор, то пространство $\operatorname{im} P \supset \operatorname{ker} X\left(\xi_{1}\right)$ конечномерно и оператор $X\left(\xi_{1}\right)$ имеет блочно-диагональный вид относительно разложения $E=\operatorname{im} P \oplus \operatorname{ker} P$ :

$$
X\left(\xi_{1}\right)=\operatorname{diag}\left(A_{0}, A_{1}\right)=\left(\begin{array}{cc}
A_{0} & 0 \\
0 & A_{1}
\end{array}\right),
$$

где оператор $A_{1}$ обратим и sp $A_{0}=\{0\}$ (см. [13, п. III.6.5]). Поэтому при любом фиксированном $\mu \in \mathbb{C} \backslash\{0\}$ оператор $X\left(\xi_{1}\right)-\mu P=\operatorname{diag}\left(A_{0}-\mu I, A_{1}\right)$ обратим на $E$, следовательно, оператор $X(\xi)-\mu P$ обратим для всех $\xi$ из некоторой окрестности $U \subset \Omega$ точки $\xi_{1}$. Имеем $X(\xi)=(X(\xi)-\mu P)\left(I+(X(\xi)-\mu P)^{-1} \mu P\right)$, где оператор $(X(\xi)-\mu P)^{-1} \mu P$ конечномерен и голоморфно зависит от $\xi \in U$. Поэтому оператор $X(\xi)$ обратим для тех и только тех значений $\xi \in U$, для которых $\tau_{U}(\xi) \neq 0$, где $\tau_{U}(\xi)=\operatorname{det}\left(I+(X(\xi)-\mu P)^{-1} \mu P\right)$ - голоморфная функция на $U$, определяемая, как в [13, п. III.4.3]. Если $\tau_{U}(\xi) \equiv 0$ в $U$, то множество тех точек $\xi \in \Omega$, в окрестностях которых $X(\xi)$ не обратим, оказывается непустым, открытым и замкнутым, т. е. совпадает со всем $\Omega$ вопреки условию обратимости $X\left(\xi_{0}\right)$. Поэтому $\tau_{U}(\xi) \not \equiv 0$ в $U$. Заметим, что $\xi \mapsto \tau_{U}(\xi) X(\xi)^{-1}-$ голоморфное отображение $U \rightarrow \mathcal{B}(E)$. Далее, если окрестности $U_{1}, U_{2}$ точек $\xi_{1}, \xi_{2} \in Z$ имеют непустое пересечение, то отношение $\tau_{U_{1}} / \tau_{U_{2}}$ голоморфно и отлично от нуля всюду на $U_{1} \cap U_{2}$. В силу классических результатов о разрешимости второй проблемы Кузена (см., например, [14, гл. IV, §16, п. 40, теорема 4]) найдется функция $\tau \in \mathcal{O}(\Omega)$ такая, что $\tau\left(\xi_{0}\right)=1$ и отношение $\tau_{U} / \tau$ голоморфно и отлично от нуля всюду на $U$ для всех $\xi_{1} \in Z$. Лемма доказана.

Допустим теперь, что $0 \leqslant \alpha<1 / m$. Тогда можно считать, что $\varphi_{0} \in G_{\alpha}\left(A_{0}\right)$ для всех $A_{0}>0$ (иначе заменим $\alpha$ на любое $\alpha_{1}, \alpha<\alpha_{1}<1 / m$, и воспользуемся леммой 3). Пусть даны произвольные числа $A, R>0$. Положим $B(R):=$ $1+\sup _{\xi \in D_{R}}\left(e m\left|p_{m}(\xi)\right|\right)^{1 / m}, A_{1}:=A, A_{2}:=A+B$ и $A_{0}:=A+2 B$. Рассуждая, как в доказательстве леммы 7 , мы видим, что семейство операторов $Y(\xi):=$ $T_{-P(\xi, \cdot)} M^{-1} K_{P(\xi, \cdot)}$ удовлетворяет условиям леммы 8 для $\Omega=D_{R}, E=G_{\alpha}(A)$, $\xi_{0}=0$. Обозначим через $\tau_{R}$ функцию из заключения леммы 8 . Тогда решение

$$
\begin{gathered}
\gamma_{-}(\xi, \cdot)=I+\left(I+T_{-P(\xi, \cdot)} M^{-1} K_{P(\xi, \cdot)}\right)^{-1} T_{-P(\xi, \cdot)} M^{-1} v_{P(\xi, \cdot)}, \\
\gamma_{+}(\xi, z)=\gamma_{-}(\xi, z) e^{P(\xi, z)} f^{-1}(z)
\end{gathered}
$$

задачи Римана (7) определено при всех $\xi \in D_{R}$, для которых $\tau_{R}(\xi) \neq 0$, а отображения $\gamma_{-}-I: D_{R} \rightarrow G_{\alpha}(A), \gamma_{+}: D_{R} \rightarrow E_{m}(B(R))$ являются мероморфными на $D_{R}$ со знаменателем $\tau_{R}(\xi)$. Как и в конце доказательства леммы 8 , мы можем решить вторую проблему Кузена и найти такую функцию $\tau \in \mathcal{O}\left(\mathbb{C}^{N}\right)$, 
что $\tau_{R} / \tau$ - голоморфная функция без нулей на $D_{R}$ для любого $R>0$. Полагая $\tau_{f}(\xi):=\tau(\xi) / \tau(0)$, завершаем доказательство утверждения (В) теоремы 3.

\section{§ 6. Формальные калибровочные преобразования}

В этом параграфе будет показано, что формула (5) задает корректно определенное инъективное отображение $L: \mathcal{O}\left(x_{0}\right)^{\text {od }} \rightarrow \mathrm{Gev}_{1}^{\text {od }}$, при этом результаты из $§ 4,5$ не используются.

Рассмотрим понятие калибровочного преобразования формальных плоских связностей. Под связностъю в данном контексте понимается любой формальный ряд Лорана вида

$$
U(x, z)=a z+\sum_{k=0}^{\infty} u_{k}(x) z^{-k}
$$

где $a \in \operatorname{gl}(n, \mathbb{C})$ - фиксированная диагональная матрица с простым спектром (см. $\S 3)$, а $u_{k} \in \mathcal{O}\left(x_{0}\right)$ для всех $k \geqslant 0$. Группа калибровочных преобразований состоит из формальных степенных рядов вида

$$
\Phi(x, z)=I+\sum_{k=0}^{\infty} \varphi_{k}(x) z^{-(k+1)},
$$

где $\varphi_{k} \in \mathcal{O}\left(x_{0}\right)$ для всех $k \geqslant 0$. Групповой операцией является обычное умножение формальных степенных рядов. По определению действие элемента $\Phi$ переводит связность $U$ в связность $V:=\Phi_{x} \Phi^{-1}+\Phi U \Phi^{-1}$. Можно еще сформулировать это определение в виде дифференциального уравнения $\Phi_{x}=V \Phi-\Phi U$ или равенства $\partial_{x}-V=\Phi\left(\partial_{x}-U\right) \Phi^{-1}$ дифференциальных операторов первого порядка. Из последнего равенства легко вывести групповое свойство: если $\Phi$ переводит $U$ в $V$, а $\Psi$ переводит $V$ в $W$, то действие ряда $\Psi \Phi$ переводит $U$ в $W$, а действие обратного ряда $\Phi^{-1}$ переводит $V$ в $U$.

Можно рассматривать калибровочное преобразование как замену неизвестной функции в уравнении $\left(\partial_{x}-U\right) \xi=0$. Переход от неизвестной функции $\xi$ к $\eta=\Phi \xi$ переводит решения системы $\left(\partial_{x}-U\right) \xi=0$ в решения системы $\left(\partial_{x}-V\right) \eta=0$, так как $\left(\partial_{x}-V\right) \eta=\Phi\left(\partial_{x}-U\right) \xi$ для всех векторнозначных функций $\xi$. Поскольку система $\left(\partial_{x}-U_{0}\right) \xi=0$ с диагональной матрицей $U_{0}$ может быть решена в явном виде, естественно найти те калибровочные преобразования, с помощью которых данная связность $U$ получается из некоторой диагональной связности $U_{0}$. Следующая лемма описывает все такие преобразования для случая, когда $U=a z+q(x), q \in \mathcal{O}\left(x_{0}\right)^{\text {od }}$. В ней (и далее) через $X_{\mathrm{d}}$ обозначается диагональная часть матрицы $X \in \operatorname{gl}(n, \mathbb{C})$ (т. е. имеем $\left(X_{\mathrm{d}}\right)_{j k}=\delta_{j k} X_{j k}$ для всех $\left.1 \leqslant j, k \leqslant n\right)$, а через $X_{\mathrm{od}}:=X-X_{\mathrm{d}}$ обозначается внедиагональная часть.

Лемма 9. Пусть $q \in \mathcal{O}\left(x_{0}\right)^{\text {od }}$. Тогда для любого набора диагональных ростков $\varkappa_{j} \in \mathcal{O}\left(x_{0}\right), j=1,2, \ldots$, и любого набора диагональньх матрии, $C_{j} \in \operatorname{gl}(n, \mathbb{C}), j=1,2, \ldots$, существует единственный формальный степенной 
ряд вида $\Phi(x, z)=I+\sum_{j=1}^{\infty} \varphi_{j}(x) z^{-j}$, где $\varphi_{j} \in \mathcal{O}\left(x_{0}\right), j=1,2, \ldots$, удовлетворяющий дифференииальному уравнению

$$
\partial_{x} \Phi(x, z)=(a z+q(x)) \Phi(x, z)-\Phi(x, z)\left(a z+\sum_{k=1}^{\infty} \varkappa_{k}(x) z^{-k}\right)
$$

и нормировочному условию $\left\{\varphi_{j}\left(x_{0}\right)\right\}_{\mathrm{d}}=C_{j}$ при $j=1,2, \ldots$.

ДокАЗАТЕльство. Сравнение коэффициентов при степенях $z$ в левой и правой частях $(13)$ дает равенства $0=\left[a, \varphi_{1}\right]+q\left(\right.$ при $\left.z^{0}\right)$ и $\varphi_{j}^{\prime}=\left[a, \varphi_{j+1}\right]+q \varphi_{j}-$ $\sum_{k=1}^{j-1} \varphi_{k} \varkappa_{j-k}-\varkappa_{j}$ (при $\left.z^{-j}, j \geqslant 1\right)$. Полагая $D_{j}:=\left(\varphi_{j}\right)_{\mathrm{d}}$ и $N_{j}:=\left(\varphi_{j}\right)_{\mathrm{od}}$, запишем эти равенства в виде

$$
\begin{gathered}
0=\left[a, N_{1}\right]+q, \\
D_{j}^{\prime}=\left(q N_{j}\right)_{\mathrm{d}}-\sum_{k=1}^{j-1} D_{k} \varkappa_{j-k}-\varkappa_{j}, \\
N_{j}^{\prime}=\left[a, N_{j+1}\right]+q D_{j}+\left(q N_{j}\right)_{\mathrm{od}}-\sum_{k=1}^{j-1} N_{k} \varkappa_{j-k} .
\end{gathered}
$$

Заметим, что линейный оператор $\mathcal{A} X:=[a, X]$ обратим на векторном пространстве всех внедиагональных матриц $X \in \operatorname{gl}(n, \mathbb{C})$. Это позволяет нам последовательно найти (т. е. выразить через $q, \varkappa_{j}$ и $C_{j}$ ) ростки $N_{1}(x)=-\mathcal{A}^{-1} q(x)$ из $\left(14_{\text {od }}^{0}\right), D_{1}(x)=C_{1}+\int_{x_{0}}^{x}\left(\left(q(s) N_{1}(s)\right)_{\mathrm{d}}-\varkappa_{1}(s)\right) d s$ из $\left(14_{\mathrm{d}}^{1}\right), N_{2}=\mathcal{A}^{-1}\left(N_{1}^{\prime}-\right.$ $\left.q D_{1}-\left(q N_{1}\right)_{\text {od }}\right)$ из $\left(14_{\text {od }}^{1}\right), D_{2}$ из $\left(14_{\mathrm{d}}^{2}\right), N_{3}$ из $\left(14_{\text {od }}^{2}\right)$ и т. д. Лемма доказана.

В лемме 9 можно выбрать $\varkappa_{j} \equiv 0$ и $C_{j}=0$ для всех целых $j \geqslant 1$. Получаем, что для любого $q \in \mathcal{O}\left(x_{0}\right)^{\text {od }}$ уравнение $m_{x}=(a z+q(x)) m-$ maz имеет единственное решение вида $m(x, z)=I+\sum_{k=0}^{\infty} m_{k}(x) z^{-(k+1)}$, где $m_{k} \in \mathcal{O}\left(x_{0}\right)$ для всех $k \geqslant 0$, такое, что ряд $m\left(x_{0}, z\right)-I$ внедиагонален. Следовательно, формула (5) сопоставляет каждому голоморфному внедиагональному ростку $q(x)$ в точке $x_{0}$ корректно определенный формальный внедиагональный степенной ряд $L q(z)$.

Лемма 10. Имеем $L q \in \mathrm{Gev}_{1}$ для всех $q \in \mathcal{O}\left(x_{0}\right)^{\text {od }}$.

ДокАзАтЕЛьство. Посмотрим на равенства (14), эквивалентные уравнению (13), с другой точки зрения. А именно, положим $D_{j} \equiv 0$ для всех целых $j \geqslant 1$ и последовательно найдем $N_{1}=-\mathcal{A}^{-1} q$ из $\left(14_{\mathrm{od}}^{0}\right), \varkappa_{1}=\left(q N_{1}\right)_{\mathrm{d}}$ из $\left(14_{\mathrm{d}}^{1}\right)$, $N_{2}=\mathcal{A}^{-1}\left(N_{1}^{\prime}-\left(q N_{1}\right)_{\text {od }}\right)$ из $\left(14_{\text {od }}^{1}\right), \varkappa_{2}=\left(q N_{2}\right)_{\text {d }}$ из $\left(14_{\mathrm{d}}^{2}\right), N_{3}$ из $\left(14_{\text {od }}^{2}\right)$ и т. д. Получаем, что для любого $q \in \mathcal{O}\left(x_{0}\right)^{\text {od }}$ существует единственное калибровочное преобразование $M(x, z)$ некоторой диагональной связности (в наших обозначениях равной $a z+\varkappa(x, z)$, где $\left.\varkappa(x, z):=\sum_{j=1}^{\infty} \varkappa_{j}(x) z^{-j}\right)$ в связность $a z+q(x)$ такое, что формальный степенной ряд $M(x, z)-I$ внедиагонален для всех $x$ из той окрестности точки $x_{0}$, в которой определен росток $q$. Покажем, что

$$
L q(z)=M\left(x_{0}, z\right)-I .
$$

Действительно, формула $\mu(x, z):=\exp \int_{x_{0}}^{x} \varkappa(s, z) d s$ задает диагональное калибровочное преобразование связности $U_{0}(x, z) \equiv a z$ в связность $a z+\varkappa(x, z)$. 
Поэтому $\Delta(x, z):=\mu^{-1}(x, z) M^{-1}(x, z) m(x, z)$ - калибровочное преобразование связности $U_{0}(x, z) \equiv a z$ в себя, а все такие преобразования диагональны и не зависят от $x$ (это вытекает из уравнений $(14)$ с $q \equiv 0$ ). Из равенства $m=M \mu \Delta=\mu \Delta+(M-I) \mu \Delta$ в силу диагональности $\mu \Delta$ и внедиагональности $M-I$ вытекает, что $m_{\mathrm{d}}=\mu \Delta$ и $m_{\mathrm{od}}=(M-I) \mu \Delta$. Отсюда получаем равенство $m_{\mathrm{od}}=(M-I) m_{\mathrm{d}}$, которое при $x=x_{0}$ преобразуется в $(15)$.

Итак, осталось установить, что $M\left(x_{0}, \cdot\right)-I \in \mathrm{Gev}_{1}$. Рассмотрим внедиагональный степенной ряд $N(x, z):=M(x, z)-I$. Уравнение $(13)$ для $M(x, z)$, имеющее вид $M_{x}=(a z+q) M-M(a z+\varkappa)$, переписывается в терминах $N(x, z)$ следующим образом: $N_{x}=z \mathcal{A} N+q(I+N)-(I+N) \varkappa$. Взяв диагональные части, получим $0=(q N)_{\mathrm{d}}-\varkappa$. Подставляя теперь $\varkappa=(q N)_{\mathrm{d}}$ во внедиагональные части, получим уравнение, которому удовлетворяет $N$ :

$$
N_{x}=z \mathcal{A} N+q+(q N)_{\text {od }}-N(q N)_{\mathrm{d}}
$$

K этому уравнению применима теорема Сибуя о жевреевской асимптотике решений сингулярно возмущенных систем (см. [15, теорема А.5.4.1], где следует заменить $\lambda$ на $z$, а $x$ на $x-x_{0}$, взять $\sigma=1$, записать все компоненты матрицы $N$ в один вектор $y$ и воспользоваться обратимостью оператора $\mathcal{A}$ ). Согласно указанной теореме имеем $N(x, \cdot) \in \mathrm{Gev}_{1}$ для всех $x$ в окрестности точки $x_{0}$. Лемма доказана.

Лемма 11. Отображение $L: \mathcal{O}\left(x_{0}\right)^{\text {od }} \rightarrow \mathrm{Gev}_{1}^{\text {od }}$ инбективно.

ДокАЗАтЕльство. Запишем

$$
q(x)=\sum_{j=0}^{\infty} q_{j}\left(x-x_{0}\right)^{j}, \quad m(x, z)=I+\sum_{j, k=0}^{\infty} m_{j k}\left(x-x_{0}\right)^{j} z^{-(k+1)} .
$$

Сравнение коэффициентов при $\left(x-x_{0}\right)^{j} z^{0}$ в равенстве $m_{x}=[a z, m]+q m$ дает набор уравнений

$$
\left[a, m_{j 0}\right]+q_{j}=0, \quad j \geqslant 0,
$$

а сравнение коэффициентов при $\left(x-x_{0}\right)^{j} z^{-(k+1)}$ в том же равенстве дает

$$
(j+1) m_{j+1, k}=\left[a, m_{j, k+1}\right]+\sum_{s=0}^{j} q_{s} m_{j-s, k}, \quad j, k \geqslant 0 .
$$

Допустим, что известны коэффициенты $m_{0 k} \in \operatorname{gl}(n, \mathbb{C})$ формального ряда $L q(z)=\sum_{k=0}^{\infty} m_{0 k} z^{-(k+1)}$. Задача состоит в том, чтобы выразить через них матрицы $m_{j 0} \in \operatorname{gl}(n, \mathbb{C})$, в терминах которых можно найти $q_{j}=\left[m_{j 0}, a\right]$ согласно (16). На первом шаге мы находим $q_{0}=\left[m_{00}, a\right]$ из $(16)$ и замечаем, что равенства (17) при $j=0$ выражают набор всех $m_{1 k}$ через набор всех $m_{0 k}$ и $q_{0}$. Это позволяет нам на втором шаге найти $q_{1}=\left[m_{10}, a\right]$ из $(16)$ и выразить все $m_{2 k}$ через уже известные матрицы $m_{1 k}, m_{0 k}, q_{0}, q_{1}$ посредством равенств (17) при $j=1$. Продолжая таким образом, получим явные формулы для всех коэффициентов $q_{j}$ ростка $q(x)$ через коэффициенты $m_{0 k}$ формального ряда $L q(z)$, чем и доказана инъективность отображения $L$. 


\section{§ 7. Доказательство теоремы 1}

Для произвольного формального степенного ряда $\varphi \in \mathrm{Gev}_{1}$ (не обязательно внедиагонального) рассмотрим задачу Римана (7), полагая $N=m=1$, $\xi=x-x_{0} \in \mathbb{C}, P(\xi, z)=a \xi z$ и $f(z)=I+\varphi(z) . \quad$ По теореме $3,(\mathrm{~A})$ решение $\gamma_{ \pm}\left(x-x_{0}, z\right)$ этой задачи существует при $x-x_{0} \in \Omega(f)$ и голоморфно зависит от $x$. В частности, все коэффициенты $g_{k}(x)$ разложения $\gamma_{-}\left(x-x_{0}, z\right)=I+\sum_{k=0}^{\infty} g_{k}(x) z^{-(k+1)}$ принадлежат $\mathcal{O}\left(x_{0}\right)$. Определим отображение $B: \mathrm{Gev}_{1} \rightarrow \mathcal{O}\left(x_{0}\right)^{\text {od }}$ формулой

$$
B \varphi(x):=\left[g_{0}(x), a\right], \quad x-x_{0} \in \Omega(f) .
$$

Это отображение является матричным нелинейным обобщением преобразования Бореля из (3). Его связь с $L$ описывается следующей леммой.

ЛЕмма 12. Имеем $L B \varphi=\varphi$ для всех $\varphi \in \mathrm{Gev}_{1}^{\text {od }}$.

ДокАЗАТЕЛЬСтво. Запишем определение рядов $\gamma_{ \pm}\left(x-x_{0}, z\right)$ в виде

$$
\gamma_{-}\left(x-x_{0}, z\right) e^{a\left(x-x_{0}\right) z} f^{-1}(z)=\gamma_{+}\left(x-x_{0}, z\right)
$$

и продифференцируем по $x$, следуя обычным правилам (которые применимы, так как обе части равенства являются голоморфными функциями от $x$ со значениями в банаховом пространстве $G_{1}(A) \oplus E_{1}(B)$, а все операции умножения суть непрерывные билинейные формы по лемме 1$)$. Получим равенство $\partial_{x} \gamma_{+}=\left(\partial_{x} \gamma_{-}+\gamma_{-} a z\right) e^{a\left(x-x_{0}\right) z} f^{-1}(z)$, при умножении которого справа на $\gamma_{+}^{-1}$ имеем $\left(\partial_{x} \gamma_{+}\right) \gamma_{+}^{-1}=\left(\partial_{x} \gamma_{-}\right) \gamma_{-}^{-1}+\gamma_{-} a z \gamma_{-}^{-1}$. Сравнение проекций левой и правой частей на $G_{1}(A) \oplus\{0\}$ и $\{0\} \oplus E_{1}(B)$ дает

$$
\left(\partial_{x} \gamma_{+}\right) \gamma_{+}^{-1}=\left\{\gamma_{-} a z \gamma_{-}^{-1}\right\}_{+}, \quad 0=\left(\partial_{x} \gamma_{-}\right) \gamma_{-}^{-1}+\left\{\gamma_{-} a z \gamma_{-}^{-1}\right\}_{-} .
$$

Поскольку $\left\{\gamma_{-} a z \gamma_{-}^{-1}\right\}_{+}=a z+B \varphi$, эти равенства принимают вид

$$
\partial_{x} \gamma_{+}=(a z+B \varphi) \gamma_{+}, \quad \partial_{x} \gamma_{-}=(a z+B \varphi) \gamma_{-}-\gamma_{-} a z .
$$

В частности, формальный степенной ряд $\gamma_{-}\left(x-x_{0}, z\right)=I+\sum_{k=0}^{\infty} g_{k}(x) z^{-(k+1)}$ удовлетворяет дифференциальному уравнению (13) для $q=B \varphi$ и $\varkappa_{j} \equiv 0$ при всех $j$. Пусть $m(x, z)$ - калибровочное преобразование из определения (5) формального степенного ряда $L q(z)$. Тогда $\Delta(x, z):=\gamma_{-}^{-1}\left(x-x_{0}, z\right) m(x, z)$ является калибровочным преобразованием связности $U_{0}(x, z) \equiv a z$ в себя, а все такие преобразования диагональны и не зависят от $x$ (это вытекает из уравнений $(14)$ для $q \equiv 0)$. Заметим, что $\gamma_{-}(0, z)=f(z)$ (действительно, при $x=x_{0}$ уравнение (19) принимает вид $\gamma_{-}(0, z) f^{-1}(z)=\gamma_{+}(0, z)$, где левая часть принадлежит $I+G_{1}(A)$, а правая принадлежит $E_{1}(B)$, следовательно, обе части равны $\left.I\right)$. Поэтому равенство $\gamma_{-}^{-1}\left(x-x_{0}, z\right) m(x, z)=\Delta(x, z)$ при $x=x_{0}$ принимает вид

$$
I+L q(z)=(I+\varphi(z)) \Delta(z)
$$

для некоторого диагонального степенного ряда $\Delta(z)$. Отделяя здесь диагональные и внедиагональные части и пользуясь (впервые в этом доказательстве) внедиагональностью $\varphi(z)$, мы получаем равенства $I=\Delta(z)$ и $L q(z)=\varphi(z) \Delta(z)$, влекущие требуемое равенство $L q(z)=\varphi(z)$. Лемма доказана. 
Из лемм 11, 12 получаем, что $L$ и $B$ суть обратные друг к другу биективные отображения между множествами $\mathcal{O}\left(x_{0}\right)^{\text {od }}$ и $\mathrm{Gev}_{1}^{\text {od }}$. Этим доказана теорема $1,(\mathrm{~A})$ и установлено равенство $B L q=q$ для всех $q \in \mathrm{Gev}_{1}^{\text {od }}$. Из этого равенства согласно теореме $3,(\mathrm{~B})$ и определению (18) отображения $B$ вытекает теорема $1,(\mathrm{~B})$.

\section{§ 8. Доказательство теоремы 2}

(А) Единственность локального голоморфного решения $q(x, t)$ задачи Коши $q\left(x, t_{0}\right)=q_{0}(x)$ для уравнения (6) вытекает из того, что уравнения, полученные из (6) $l$-кратным дифференцированием обеих частей по $x$, однозначно выражают все частные производные $\partial_{t}^{k} \partial_{x}^{l} q\left(x_{0}, t_{0}\right)$ через производные функции $q_{0}(x)$ в точке $x=x_{0}$. Необходимость условия $L q_{0} \in \mathrm{Gev}_{1 / m}$ для существования локального голоморфного решения установлена в [9, теорема 4]. Чтобы доказать достаточность, возьмем произвольный росток $q_{0} \in \mathcal{O}\left(x_{0}\right)^{\mathrm{od}}, L q_{0} \in \mathrm{Gev}_{1 / m}$, и рассмотрим задачу Римана (7) при $N=2, \xi=\left(x-x_{0}, t-t_{0}\right) \in \mathbb{C}^{2}$, $P(\xi, z)=a z\left(x-x_{0}\right)+\left(b z^{m}+c_{1} z^{m-1}+\cdots+c_{m}\right)\left(t-t_{0}\right)$ и $f(z)=I+L q_{0}(z)$. По теореме $3,(\mathrm{~A})$ решение $\gamma_{ \pm}\left(x-x_{0}, t-t_{0}, z\right)$ этой задачи существует в некоторой окрестности точки $\left(x_{0}, t_{0}\right) \in \mathbb{C}^{2}$ и голоморфно зависит от $x, t$. Положим $E(x, t, z):=\gamma_{+}\left(x-x_{0}, t-t_{0}, z\right)$ и $q(x, t):=\left[g_{0}(x, t), a\right]$, где $g_{0}(x, t)-$ коэффициент при $z^{-1}$ в разложении

$$
\gamma_{-}\left(x-x_{0}, t-t_{0}, z\right)=I+\sum_{k=0}^{\infty} g_{k}(x, t) z^{-(k+1)} .
$$

Равенство $B L q_{0}=q_{0}$ из $\S 7$ показывает, что $q\left(x, t_{0}\right)=q_{0}(x)$ для всех $x$, близких к $x_{0}$. Кроме того, из первого равенства (20) вытекает, что в окрестности точки $\left(x_{0}, t_{0}\right)$ имеем $E_{x}=U E$, где $U(x, t, z):=a z+q(x, t)$. Повторяя вывод равенств (20) с заменой $x$ на $t$, получаем, что $E_{t}=V E$, где $V(x, t, z):=\sum_{k=0}^{m} F_{m-k}(q)(x, t) z^{k}$ в обозначениях $\S 3$ (подробности этого рассуждения приведены в доказательстве теоремы 1 работы [9] для случая $L q_{0} \in \mathrm{Gev}_{0}$ и остаются справедливыми для рассматриваемого нами случая $\left.L q_{0} \in \operatorname{Gev}_{1 / m}\right)$. Из уравнений $E_{x}=U E$ и $E_{t}=V E$ и обратимости $E$ следует равенство $U_{t}-V_{x}+[U, V]=0$, эквивалентное уравнению (6).

(В) Для любого $t_{1} \in \mathbb{C},\left|t_{1}-t_{0}\right|<\delta_{2}$, положим $q_{1}(x):=q\left(x, t_{1}\right)$. Тогда $L q_{1} \in \mathrm{Gev}_{1 / m}$ по теореме 2, (А) (или по теореме 4 из работы [9]). Поскольку $1 / m \leqslant 1 / 2$, теорема $1,(\mathrm{~B})$ дает мероморфное продолжение функции $x \mapsto q\left(x, t_{1}\right)$ на все $\mathbb{C}$. Так как это верно для всех $t_{1} \in \mathbb{C},\left|t_{1}-t_{0}\right|<\delta_{2}$, то полученное продолжение $q(x, t)$ мероморфно в полосе $\left\{(x, t) \in \mathbb{C}^{2}|| t-t_{0} \mid<\delta_{2}\right\}$ как функция двух переменных. (Можно вывести последнее заключение и прямо из теоремы $3,(\mathrm{~B})$, доказательство которой остается справедливым, если один или несколько радиусов полидиска $\Omega$ обращаются в бесконечность.)

Предъявим голоморфное решение $q_{0}(x, t)$ уравнения $(6)$ в исходном бидиске, не продолжаемое ни через одну граничную точку упомянутой полосы. Для этого заметим, что правая часть уравнения (6) есть полином от $q, \partial_{x} q, \ldots, \partial_{x}^{m} q$, причем старшая производная $\partial_{x}^{m} q$ входит в этот полином только в первой степени и с постоянным ненулевым коэффициентом (см., например, [9]). Поэтому 
теорема Коши-Ковалевской гарантирует существование локальных голоморфных решений $q(x, t)$ уравнения (6) с любыми (голоморфными внедиагональными) начальными данными $\partial_{x}^{k} q\left(x_{0}, t\right)=\psi_{k}(t), 0 \leqslant k \leqslant m-1$. Выбрав функцию $\psi_{0}(t)$ голоморфно непродолжаемой ни через одну граничную точку круга $\left\{\left|t-t_{0}\right|<\delta_{2}\right\}$, получим требуемый пример.

(C) Это утверждение немедленно вытекает из теоремы $3,(\mathrm{~B})$ и описанного в доказательстве теоремы $2,(\mathrm{~A})$ построения решения $q(x, t)$ по данным рассеяния $L q_{0}(z)$ начального условия.

\section{§9. Замечания и примеры}

(А) Результат из $§ 1$ о продолжении локальных голоморфных решений не переносится на общие уравнения вида $u_{t}=P(u)$, где $P$ - дифференциальный полином по $x$. Например, для уравнения

$$
u_{t}=k u^{m} u_{x}+l u_{x x x}, \quad k, l \in \mathbb{C} \backslash\{0\}, \quad m=0,1,2, \ldots,
$$

этот результат справедлив только при $m=0$ (линейный случай), $m=1$ (уравнение Кортевега-де Фриза) и $m=2$ (модифицированное уравнение Кортевега-де Фриза). При любом целом $m>2$ это уравнение имеет решение вида $u=\beta_{m}\left(x-x_{0}\right)^{-2 / m}$ (для надлежащей константы $\beta_{m} \neq 0$ ) с точкой ветвления при $x=x_{0}$. Кроме того, обсуждаемый результат неверен как для солитонных уравнений, не представимых в виде (6), так и для линейных уравнений с более чем одной пространственной переменной. Примеры: локальная голоморфная задача Коши для уравнения $u_{t t}-u_{x x}=\sin u$ разрешима при любых начальных условиях (см. [8]), а линейное уравнение $\partial_{t} u=\left(\partial_{x_{1}}^{2}+\partial_{x_{2}}^{2}\right) u$ имеет решение $u=\ln \left(x_{1}^{2}+x_{2}^{2}\right)$ с точками ветвления по $x_{1}, x_{2}$.

(В) Теорема 1 является лишь частичным аналогом предложения 1. В общем случае едва ли можно дать простое описание множества всех $q \in \mathcal{O}\left(x_{0}\right)^{\text {od }}$ таких, что $L q \in \operatorname{Gev}_{\alpha}$ для данного $\alpha \in[0,1)$. По крайней мере условий на рост при $|x| \rightarrow \infty$ для этого заведомо не достаточно: если $q^{0}(x)$ - внедиагональная $(2 \times 2)$-матрица с $q_{12}^{0}(x)=x$ и $q_{21}^{0}(x)=1$, то $L q^{0}$ принадлежит $\operatorname{Gev}_{1 / 3}$, но не принадлежит $\mathrm{Gev}_{1 / 3-0}$. Тем не менее, следующая гипотеза весьма правдоподобна в силу предложения 1 и известных примеров эллиптических функций, удовлетворяющих уравнению Кортевега-де Фриза.

ГипотезА 1. Если $q \in \mathcal{O}\left(x_{0}\right)^{\text {od }} u L q \in \operatorname{Gev}_{\alpha}$ при некотором $\alpha \in[0,1)$, то $q(x)$ - мероморфная функция порядка не выме $1+(1-\alpha)^{-1}$ на $\mathbb{C}$.

Из доказательства леммы 12 видно, что еще одним необходимым условием для включения $L q \in \mathrm{Gev}_{1-0}$ является существование глобально мероморфной фундаментальной системы решений уравнения $E_{x}=(a z+q(x)) E$ при каждом $z \in \mathbb{C}$. В наших обозначениях эта система состоит из столбцов матрицы $\gamma_{+}(x, z)$. Одно из следствий существования такой системы решений отмечено в [9, теорема 2]. Неизвестно, является ли это необходимое условие также и достаточным хотя бы в классе рациональных $\operatorname{gl}(n, \mathbb{C})$-значных функций $q(x)$, голоморфных при $x=\infty$.

(C) Утверждение, эквивалентное теореме $1,(\mathrm{~A})$, содержится в работе [16, c. 99-103] (эквивалентность вытекает из формулы (15)) вместе с набросками 
доказательств, которые, однако, автору не удалось проследить до конца. В настоящей работе теорема $1,(\mathrm{~A})$ доказана другим способом.

(D) Под конечнозонными решениями уравнения (6) можно понимать такие решения $q(x, t)$, начальные данные которых $q_{0}(x)$ задаются не зависящим от $t$ решением какого-либо потока той же иерархии, т. е. какого-либо уравнения вида (6) с теми же матрицами $a$ и $b$ (см. [5] или [17], где показано, что в этом случае $\left.L q_{0} \in \mathrm{Gev}_{0}\right)$. Такие функции $q_{0}(x)$ задают решения всех потоков, глобально мероморфные на $\mathbb{C}^{2}$ (что вытекает также из формул для конечнозонных решений через тета-функции [5]). Однако обратное неверно: так как множество $\bigcap_{\alpha>0} \mathrm{Gev}_{\alpha}$ строго больше, чем $\mathrm{Gev}_{0}$ (достаточно взять $\left|\varphi_{n}\right|=\ln (n !)$ ), то из теорем $1,(\mathrm{~A})$ и $2,(\mathrm{C})$ вытекает существование таких мероморфных на $\mathbb{C}$ функций $q_{0}(x) \not \equiv$ const, что задача Коши $q(x, t)=q_{0}(x)$ для уравнения (6) с данной матрицей $a$ имеет глобально мероморфное решение на $\mathbb{C}^{2}$ при любом $m \geqslant 2$ (и при любом выборе матриц $\left.b, c_{1}, c_{2}, \ldots\right)$, но ни одно из этих решений не является конечнозонным.

В случае верхнетреугольных $(2 \times 2)$-матриц $q(x, t)$, когда уравнения $(6)$ сводятся к (4), все конечнозонные решения суть целые функции на $\mathbb{C}^{2}$ (экспоненциальные полиномы). Однако это уже не так для других условий симметрии. Скажем, конечнозонные решения уравнения Кортевега-де Фриза (1) всегда имеют полюсы на $\mathbb{C}^{2}$. Это позволяет выдвинуть следующую гипотезу.

ГиПотезА 2. Всякое целое решение $u \in \mathcal{O}\left(\mathbb{C}^{2}\right)$ уравнения (1) постоянно.

Непостоянные решения уравнения (1) могут быть целыми функциями от $x$ при почти всех $t$ или от $t$ при почти всех $x$. Примеры: $u_{1}(x, t)=x /(1-k t)$ и $u_{2}(x, t)=C x^{-2}$, где $C=-12 l / k$. Любопытно, что при этом локальная голоморфная задача Коши с начальным условием $u(x, 0)=u_{2}(x, 0)$ разрешима для всех потоков иерархии Кортевега-де Фриза (решение $u_{2}$ является конечнозонным), а такая же задача Коши с начальным условием $u(x, 0)=u_{1}(x, 0)$ не разрешима ни для одного из этих потоков, кроме самого уравнения (1). Последнее ясно из теоремы $2,(\mathrm{~A})$ и включения $L q^{0} \in \mathrm{Gev}_{1 / 3} \backslash \mathrm{Gev}_{1 / 3-0}$, упомянутого в замечании (B).

(Е) Если в условиях леммы 8 все операторы $Y(\xi)$ являются ядерными, то в качестве функции $\tau(\xi)$ в этой лемме можно взять $\operatorname{det}(I+Y(\xi))$. Однако для интересующих нас операторов $Y(\xi)=i_{1} T_{-P(\xi, \cdot)} M^{-1} K_{P(\xi, \cdot)} i_{1}^{-1} \in \mathcal{B}\left(l_{1}\right)$ выполнено условие $\sum_{p, q=0}^{\infty}\left|(Y(\xi))_{p q}\right|<\infty$, более сильное, чем ядерность. (Напомним, что оператор $Y \in \mathcal{B}\left(l_{1}\right)$ является ядерным тогда и только тогда, когда $\sum_{p=0}^{\infty} \sup _{q \geqslant 0}\left|Y_{p q}\right|<\infty$; см., например, [18, с. 381, формула (1)].) Это позволяет выразить $\tau(\xi)$ с помощью более простого и классического понятия определителя Хилла (см. $[19, \S 2.8,19.4])$. Поэтому в условиях теоремы $3,(\mathrm{~B})$ все коэффициенты формальных степенных рядов $\gamma_{ \pm}(\xi, z)$ суть мероморфные функции от $\xi$, имеющие общий знаменатель, который явно выражается через коэффициенты полинома $P(\xi, z)$ и степенного ряда $f(z)$ с помощью определителя Хилла. То же самое относится и к знаменателям мероморфных функций $q(x)=B \varphi(x)$ и $q(x, t)$, определенных в доказательствах теорем 1,2 . 


\section{Список литературы}

1. Э. Гурса, Курс математического анализа, т. 3, ч. 1: Бесконечно близкие интеграль. Уравнения с частными производными, ГТТИ, М.-Л., 1933; пер. с фр.: E. Goursat, Cours d'analyse mathematique, Tome III. Intégrales infiniment voisines. Équations aux dérivées du second ordre. Équations intégrales. Calcul des variations, Gauthier-Villars, Paris, 1927.

2. А. Ф. Леонтьев, Целье функиии. Рядъ экспонент, Наука, М., 1983.

3. D. V. Widder, The heat equation, Academic Press, New York-London, 1975.

4. В. Е. Захаров, С. В. Манаков, С. П. Новиков, Л. П. Питаевский, Теория солитонов. Метод обратной задачи, Наука, М., 1980; англ. пер.: S. Novikov, S. V. Manakov, L. P. Pitaevskiǔ, V. E. Zakharov, Theory of solitons, Contemp. Soviet Math., Plenum, New York-London, 1984.

5. Б. А. Дубровин, И. М. Кричевер, С.П. Новиков, "Интегрируемые системы. I", Динамические системъ-4, Итоги науки и техн. Сер. Соврем. пробл. мат. Фундам. направления, 4, ВИНИТИ, М., 1985, 179-277; англ. пер.: B. A. Dubrovin, I. M. Krichever, S. P. Novikov, "Integrable systems. I", Dynamical systems. IV. Symplectic geometry and its applications, Encyclopaedia Math. Sci., 4, Springer-Verlag, Berlin, 1990, 173-280.

6. Л. А. Тахтаджян, Л. Д. Фаддеев, Гамильтонов подход в теории солитонов, Наука, M., 1986; англ. пер.: L. D. Faddeev, L. A. Takhtadzhyan, Hamiltonian methods in the theory of solitons, Springer Ser. Soviet Math., Springer-Verlag, Berlin, 1987.

7. В. Е. Захаров, А. Б. Шабат, "Интегрирование нелинейных уравнений математической физики методом обратной задачи рассеяния. II", Функи. анализ и его прил., 13:3 (1979), 13-22; англ. пер.: V. E. Zakharov, A. B. Shabat, "Integration of the nonlinear equations of mathematical physics by the method of the inverse scattering problem. II", Functional Anal. Appl., 13:3 (1979), 166-174.

8. И. М. Кричевер, “Аналог формулы Даламбера для уравнений главного кирального поля и уравнения sine-Gordon”, Докл. АН СССР, 253:2 (1980), 288-292; англ. пер.: I. M. Krichever, "An analogue of d'Alembert's formula for the equations of the principal chiral field and for the Sine-Gordon equation", Soviet Math. Dokl., 22:1 (1980), 79-84.

9. А. В. Домрин, “Замечания о локальном варианте метода обратной задачи рассеяния", Комплексный анализ и приложения, Тр. МИАН, 253, Наука, М., 2006, 46-60; англ. пер.: A.V. Domrin, "Remarks on the local version of the inverse scattering method", Proc. Steklov Inst. Math., 253:1 (2006), 37-50.

10. С. П. Новиков, "Периодическая задача для уравнения Кортевега-де Фриза. I", Функи. анализ и его прил., 8:3 (1974), 54-66; англ. пер.: S. P. Novikov, "The periodic problem for the Korteweg-de Vries equation", Functional Anal. Appl., 8:3 (1974), 236-246.

11. И. Ц. Гохберг, "О линейных операторах, аналитически зависящих от параметра", Докл. АН СССР, 78:4 (1951), 629-632.

12. M. Ribarič, I. Vidav, "Analytic properties of the inverse $A(z)^{-1}$ of an analytic linear operator valued function A(z)", Arch. Rational Mech. Anal., 32:4 (1969), 298-310.

13. Т. Като, Теория возмущений линейных операторов, Мир, М., 1972; пер. с англ.: Т. Kato, Perturbation theory for linear operators, Springer-Verlag, Berlin-Heidelberg-New York, 1966.

14. Б. В. Шабат, Введение в комплексный анализ, т. II. Функиии нескольких переменных, Наука, М., 1985; фр. пер.: B. Chabat, Introduction á l'analyse complexe, T. 2. Fonctions de plusieurs variables, Traduit Russe Math., Mir, Moscow, 1990.

15. Ya. Sibuya, Linear differential equations in the complex domain: problems of analytic continuation, Transl. Math. Monogr., 82, Amer. Math. Soc., Providence, RI, 1990. 
16. M. U. Schmidt, "Integrable systems and Riemann surfaces of infinite genus", Memoirs Amer. Math. Soc., 122:581 (1996).

17. А.В. Домрин, "Задача Римана и матричнозначные потенциалы со сходящейся функцией Бейкера-Ахиезера", ТМФ, 144:3 (2005), 453-471; англ. пер.: A. V. Domrin, "The Riemann problem and matrix-valued potentials with a convergent Baker-Akhiezer function", Theoret. and Math. Phys., 144:3 (2005), 1264-1278.

18. A. Grothendieck, "La théorie de Fredholm", Bull. Soc. Math. France, 84 (1956), 319-384.

19. Э. Т. Уиттекер, Дж. Н. Ватсон, Курс современного анализа, Физматлит, М., 1963; пер. с англ. Е. T. Whittaker, G. N. Watson, A course of modern analysis, Cambridge Univ. Press, Cambridge, 1927.

A. В. Домрин (A. V. Domrin)

Поступило в редакцию

Московский государственный университет

31.03.2008

им. М. В. Ломоносова

E-mail: domrin@mi.ras.ru 